Topologia de singularidades e o estudo de seus invariantes. 


\title{
Topologia de singularidades e o estudo de seus invariantes.1
}

\author{
Grazielle Feliciani Barbosa \\ Orientador: Prof. Dr. Marcelo José Saia \\ Co-Orientador: Prof. Dr. Joachim Herbert Rieger
}

Tese apresentada ao Instituto de Ciências Matemáticas e de Computação da Universidade de São Paulo, como parte dos requisitos para obtenção do título de Doutora em Ciências Área: Matemática.

$$
\begin{gathered}
\text { USP - São Carlos } \\
\text { Março/2008 }
\end{gathered}
$$

\footnotetext{
${ }^{1}$ Este trabalho teve suporte financeiro da Capes
} 
Ao Mauricio com imenso amor ... 


\section{Agradecimentos}

A Deus, meu eterno companheiro, que esteve ao meu lado em todos os momentos.

Agradeço aos professores Marcelo José Saia e Joachim Herbert Rieger pela orientação, pela paciência, pela dedicação e, principalmente, pela amizade. Agradeço ao professor Joachim pela acolhida e atenção que me dedicou quando estive em Halle. Agradeço à professora Roberta pela dedicação, por ter ido comigo para Alemanha, por me ouvir e por acreditar em mim mesmo quando eu não acreditava. Você será sempre minha orientadora. Obrigada!

Agradeço aos meus pais, José Lourenço e Inês, por todo amor e carinho que me dedicam, por terem me ensinado o caminho a seguir e por acreditarem em mim. À minha irmã Giselle, agradeço pela amizade e compreensão. Agradeço à toda minha família, pelas orações que me dispensaram e por me amarem do jeito que eu sou. Amo vocês!

Ao meu namorado Mauricio, agradeço por estar sempre ao meu lado, com muita dedicação, carinho e amor. Te amo coração...

Agradeço a Deus todos os dias pelos amigos que me deu, não são muitos, nem poucos, mas são especiais para mim... e eu amo cada um de vocês.

A todos os professores que fizeram parte da minha vida acadêmica e sempre me incentivaram a prosseguir em meus estudos sem desistir de meus objetivos. Agradeço em especial à professora Maria Aparecida Soares Ruas.

Aos funcionários do ICMC por toda atenção dispensada e por serem pessoas tão gentis.

À Capes pelo suporte financeiro.

Enfim, a todos aqueles que colaboraram para a realização deste trabalho. 


\section{Resumo}

Algumas relações entre $\mathcal{A}$-invariantes de germes de aplicações de coposto 1 equidimensionais $f: \mathbb{C}^{n}, 0 \rightarrow \mathbb{C}^{n}, 0$ são descritas. O principal resultado estabelece que a soma alternada de números de Milnor dos fechos dos conjuntos $A_{i}$ na fonte de $f$ é igual a multiplicidade local de $f$ menos $n+1$. E existem fórmulas correspondentes para os $s$-tipos estáveis locais $A_{\left(k_{1}, \ldots, k_{s}\right)}$. As relações nos garantem condições para a $\mathcal{A}$-finitude de $f$ e para a $\mathcal{A}$-trivialidade topológica de deformações de $f$. Também classificamos os germes de aplicações $\mathcal{A}$-simples $f: \mathbb{C}^{2}, 0 \rightarrow \mathbb{C}^{5}, 0$, para multiplicidades 1,2 e 3 . 


\section{Abstract}

Some new relations between $\mathcal{A}$-invariants of equidimensional corank-1 map-germs $f: \mathbb{C}^{n}, 0 \rightarrow \mathbb{C}^{n}, 0$ are described. The main local result states that the alternating sum of the Milnor numbers of the closures of the $A_{i}$ sets in the source of $f$ is equal to the local multiplicity of $f$ minus $n+1$. And there are corresponding formulas for the $s$-local stable types $A_{\left(k_{1}, \ldots, k_{s}\right)}$. The relations provide simplified (or weaker) conditions for the $\mathcal{A}$-finiteness of $f$ and for the topological $\mathcal{A}$-triviality of deformations of $f$. We also classify the $\mathcal{A}$-simple germs $f: \mathbb{C}^{2}, 0 \rightarrow \mathbb{C}^{5}, 0$, for multiplicities 1,2 and 3 . 


\section{Sumário}

Introdução

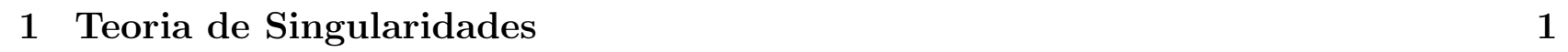

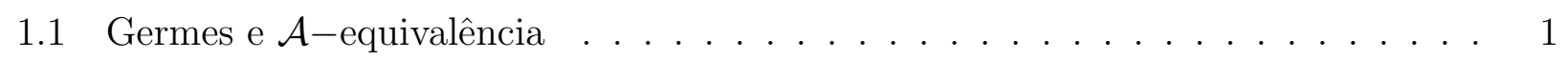

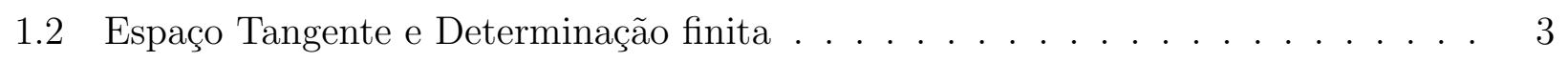

1.3 Equivalência de contato . . . . . . . . . . . . . . . . . . . . 5

1.4 Desdobramentos . . . . . . . . . . . . . . . . . . . 6

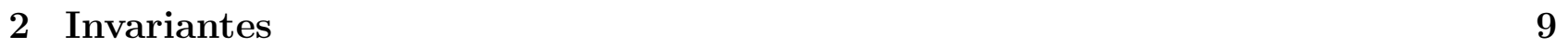

2.1 Preliminares . . . . . . . . . . . . . . . . . . . . . . . . 9

$3 \quad$ Relações entre invariantes de germes de coposto 1 de $\mathbb{C}^{n}$ em $\mathbb{C}^{n} \quad 15$

3.1 Relações entre invariantes . . . . . . . . . . . . . . . . . . 15

\begin{tabular}{|ll}
4 Aplicações & 27
\end{tabular}

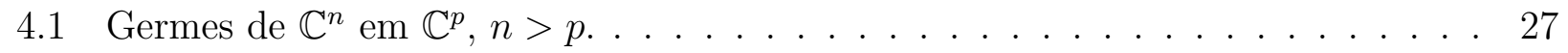

4.2 Determinação finita e equisingularidade $\ldots \ldots \ldots \ldots$. . . . . . . . . . . . . . 28

$5 \quad$ Classificação de germes de aplicações de $\mathbb{C}^{2}$ em $\mathbb{C}^{n}, n \geq 5$

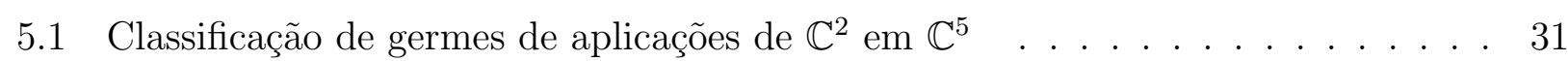

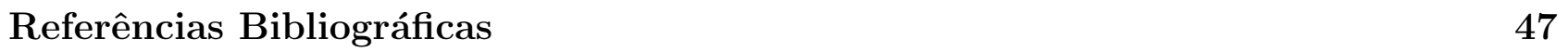




\section{Introdução}

Uma questão relevante em teoria de singularidades é o estudo dos invariantes associados a um germe de aplicação. O primeiro invariante que surgiu foi o número de Milnor de um germe de uma função analítica $f: \mathbb{C}^{n}, 0 \rightarrow \mathbb{C}, 0$. Este invariante pode ser definido de várias maneiras. Algébricamente é definido por

$$
\mu=\operatorname{dim}_{\mathbb{C}} \frac{\mathcal{O}_{n}}{\left\langle\frac{\partial f}{\partial x_{1}}, \frac{\partial f}{\partial x_{2}}, \ldots, \frac{\partial f}{\partial x_{n}}\right\rangle}
$$

Sua finitude é uma condição necessária e suficiente para a determinação finita e, além disso, ele aparece associado à geometria da singularidade em duas formas: como números de pontos críticos de Morse em uma deformação estável e como o posto da homologia média da fibra de Milnor de $f$. Na teoria de singularidades de germes de aplicações $f: \mathbb{C}^{n}, 0 \rightarrow \mathbb{C}^{p}, 0$, com $p>1$, não encontramos um invariante assim tão completo. Uma razão para isto está no fato de que, enquanto para funções aparece apenas um tipo de singularidade estável, ou seja, a singularidade de Morse, para $p>1$ a complexidade da classificação dos germes e multigermes estáveis aumenta com $n$ e $p$.

As singularidades de germes de aplicações do plano no plano foram primeiramente estudadas por H. Whitney, em 1955 e, posteriormente, por J. Mather. Os trabalhos de Whitney e Mather versam sobre singularidades estáveis. A classificação dos germes simples de coposto 1 ( caso real ou complexo ) foi obtida por J. H. Rieger em [25].

Quando um germe finitamente determinado não estável de $f: \mathbb{C}^{2}, 0 \rightarrow \mathbb{C}^{2}, 0$ é perturbado de modo a se tornar estável, um certo número de cúspides e dobras aparecem no discriminante, que é uma curva. Na tabela 1 em [25] alguns invariantes foram calculados para cada germe simples $f$, por exemplo, o número de cúspides $c(f)$, o número de dobras $d(f)$, a multiplicidade local $m_{f}(0)$ e o número de Milnor $\mu\left(\Sigma_{f}\right)$ do conjunto crítico. Também mostrou que alguns desses invariantes satisfazem certas relações, por exemplo, temos $c(f)=\mu\left(\Sigma_{f}\right)+m_{f}(0)-2$. Gaffney e Mond mostraram em [11] que esta formula também vale para germes de coposto 2 ( o que havia sido conjecturado por J. Rieger, baseado em seu trabalho com M.A.S. Ruas sobre a classificação de germes simples de coposto 2 no plano [29]). 
Para germes quase-homogêneos equidimensionais de coposto 1, Marar, Montaldi e Ruas [22] apresentaram fórmulas para os invariantes 0-estáveis em termos dos pesos e grau de $f$. Mais geralmente, usando as equções que definem o fecho do conjunto dos pontos do tipo $A_{\left(k_{1}, \ldots, k_{s}\right)}$, podemos obter fórmulas para os invariantes 0-estáveis $r_{\left(k_{1}, \ldots, k_{s}\right)}(f)$, onde $\sum_{i} k_{i}=n$, de qualquer germe de coposto 1 equidimensional $f$, como em [26]. Fórmulas relacionando os invariantes 0-estáveis $r_{\left(k_{1}, . ., k_{s}\right)}(f)$, a multiplicidade de $f$ e certos números de Milnor generalizam, por exemplo, a fórmula $c(f)=\mu\left(\Sigma_{f}\right)+m_{f}(0)-2$ para $n=2($ provada para o caso semi- quase-homogêneo em 27.

Nos capítulos 2 a 4 desta tese (baseados em um trabalho em conjunto com Rieger, ver [3]) provamos essas fórmulas no caso geral (sem a hipótese de semi-quase-homogeneidade) e aplicamos estas à problemas de determinação finita e equisingularidade.

Também apresentamos neste trabalho a classificação de germes $\mathcal{A}$-simples $f: \mathbb{C}^{2}, 0 \rightarrow \mathbb{C}^{5}, 0$, para multiplicidades 1, 2 e 3, e uma conjectura de Rieger para generalizar essa classificação.

No capítulo 1 apresentamos algumas definições e resultados da Teoria de Singularidades que são de grande importância para o ínicio do trabalho

O objetivo do capítulo 2 é apresentar os invariantes $v$ e $r$ relacionados a um germe de aplicação equidimensional de coposto 1. Apresentamos também a fórmula de Lê-Greuel, de grande importância para obtenção dos resultados apresentados no capítulo 3, e demonstramos um lema sobre aditividade de codimensões.

No capítulo 3 apresentamos relações entre $\mathcal{A}$-invariantes de germes de aplicações de coposto 1 equidimensionais. O principal resultado estabelece que a soma alternada de números de Milnor dos fechos dos conjuntos $A_{i}$ na fonte de $f$ é igual a multiplicidade local de $f$ menos $n+1$. E existem fórmulas correspondentes para os $s$-tipos estáveis locais $A_{\left(k_{1}, \ldots, k_{s}\right)}$.

O capítulo 4 contém algumas extensões a germes $f: \mathbb{C}^{n}, 0 \rightarrow \mathbb{C}^{p}, 0, n>p$ e aplicações à determinação finita e equisingularidade.

No capítulo 5 classificamos os germes $\mathcal{A}$-simples $f: \mathbb{C}^{2}, 0 \rightarrow \mathbb{C}^{5}, 0$, para multiplicidades 1 , 2 e 3 , e apresentamos uma conjectura para generalizar essa classificação para o caso de germes $\mathcal{A}$-simples de $\mathbb{C}^{2}, 0$ em $\mathbb{C}^{n}, 0, n \geq 5$. 


\section{Capítulo 1}

\section{Teoria de Singularidades}

Neste capítulo apresentamos alguns tópicos da teoria de singularidades necessários para o desenvolvimento deste trabalho. A principal referência é Gibson [12].

\subsection{Germes e $\mathcal{A}$-equivalência}

Seja $S \subset \mathbb{C}^{n}$. Consideremos o conjunto das aplicações analíticas definidas numa vizinhança aberta de $S$ em $\mathbb{C}^{n}$ com valores em $\mathbb{C}^{p}$. Introduzimos neste conjunto a seguinte relação de equivalência: dadas $f: U \rightarrow \mathbb{C}^{p}$ e $g: V \rightarrow \mathbb{C}^{p}$ em tal conjunto, dizemos que $f$ e $g$ são equivalentes se existe uma vizinhança aberta $W \subset U \cap V$ de $S$ tal que $\left.f\right|_{W}=\left.g\right|_{W}$.

Definição 1.1.1 Definimos o multigerme de uma aplicação holomorfa $f: U \rightarrow \mathbb{C}^{p}$, onde $U$ é uma vizinhança aberta de $S$ em $\mathbb{C}^{n}$, como a classe de equivalência de $f$ segundo a relação acima. Denotamos o multigerme de $f$ por $f: \mathbb{C}^{n}, S \rightarrow \mathbb{C}^{p}, y$. Se $S=\{x\}$, esta classe de equivalência é chamada de germe de aplicação ou, simplesmente, germe.

A derivada do germe $f: \mathbb{C}^{n}, x \rightarrow \mathbb{C}^{p}, y$ é a derivada em $x$ de qualquer um de seus representantes.

Definição 1.1.2 Dizemos que um germe $f: \mathbb{C}^{n}, x \rightarrow \mathbb{C}^{p}, y$ é um germe de difeomorfismo se um de seus representantes, e portanto todos, é um difeomorfismo local em $x$.

Definição 1.1.3 O posto de um germe $f: \mathbb{C}^{n}, x \rightarrow \mathbb{C}^{p}, y$ é definido como o posto da matriz de sua derivada em $x$ e é denotado como posto $f$. $O$ coposto do germe $f$ é definido como $\min \{n, p\}-$ posto $f$. 
Definição 1.1.4 Dizemos que dois germes $f_{1}: \mathbb{C}^{n}, x_{1} \rightarrow \mathbb{C}^{p}, y_{1}$ e $f_{2}: \mathbb{C}^{n}, x_{2} \rightarrow \mathbb{C}^{p}, y_{2}$ são $\mathcal{A}$-equivalentes, e denotamos por $f_{1} \sim_{\mathcal{A}} f_{2}$, se existem germes de difeomorfismos $\phi: \mathbb{C}^{n}, x_{1} \rightarrow \mathbb{C}^{n}, x_{2}$ e $\psi: \mathbb{C}^{p}, y_{1} \rightarrow \mathbb{C}^{p}, y_{2}$ para os quais o diagrama:

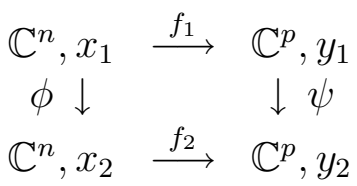

é comutativo, ou seja, $f_{2}=\psi \circ f_{1} \circ \phi^{-1}$.

Como consequência da Definição 1.1.4, todo germe $\mathbb{C}^{n}, x \rightarrow \mathbb{C}^{p}, y$ é $\mathcal{A}$-equivalente a algum germe $\mathbb{C}^{n}, 0 \rightarrow \mathbb{C}^{p}, 0$. O conjunto dos germes $\mathbb{C}^{n}, 0 \rightarrow \mathbb{C}^{p}, y$ é denotado por $\mathcal{O}_{n, p}$. Quando $p=1$, denotamos por $\mathcal{O}_{n}$. $\mathcal{O}^{n}$ é um anel local cujo ideal maximal é $\mathcal{M}_{n}=\left\{f \in \mathcal{O}^{n} / f(0)=0\right\}$. O conjunto dos germes de difeomorfismos de $\mathcal{O}_{n, n}$ é um grupo com a operação de composição e é denotado por Diff $\left(\mathbb{C}^{n}\right)$. Definimos o grupo $\mathcal{A}=\operatorname{Diff}\left(\mathbb{C}^{n}\right) \times \operatorname{Diff}\left(\mathbb{C}^{p}\right)$

Um germe $f \in \mathcal{O}_{n, p}$ induz o homomorfismo de álgebras

$$
\begin{aligned}
f^{*}: \mathcal{O}_{p} & \longrightarrow \mathcal{O}_{n} \\
h & \longmapsto h \circ f
\end{aligned}
$$

Definição 1.1.5 A multiplicidade local de um germe de aplicação $f: \mathbb{C}^{n}, 0 \rightarrow \mathbb{C}^{p}, 0$ é

$$
m_{f}(0)=\operatorname{dim}_{\mathbb{C}} \frac{\mathcal{O}_{n}}{f^{*} \mathcal{M}_{p}}
$$

Definição 1.1.6 Denotamos por $J^{k}(n, p)$ o espaço vetorial complexo das aplicações $f: \mathbb{C}^{n} \rightarrow$ $\mathbb{C}^{p}$ onde cada componente $f_{i}$ de $f$ é um polinômio de grau menor ou igual a $k$ nas coordenadas $x_{1}, \ldots, x_{n}$ com termo constante nulo. Os elementos de $J^{k}(n, p)$ são chamados $k$-jatos.

Definimos $H^{k}(n, p)$ como o espaço vetorial complexo das aplicações $f: \mathbb{C}^{n} \rightarrow \mathbb{C}^{p}$ onde cada componente $f_{i}$ de $f$ é um polinômio homogêneo de grau $k$ nas coordenadas $x_{1}, \ldots, x_{n}$.

Para cada aplicação holomorfa $f: U \subset \mathbb{C}^{n} \rightarrow \mathbb{C}^{p}$, onde $U$ é um aberto de $\mathbb{C}^{n}$ e $a \in U$, a expansão em séries de potências de $f(x+a)$ em torno da origem é dada por

$$
f(a)+d_{a} f \cdot x+\frac{1}{2 !} d_{a}^{2} f \cdot x^{2}+\cdots
$$


Definição 1.1.7 Sejam $f \in \mathcal{O}_{n, p}$ e $k \in \mathbb{Z}, k \geq 1$. O $k$-jato de $f$ em a, denotado por $j^{k} f(a)$, é definido por

$$
d_{a} f \cdot x+\frac{1}{2 !} d_{a}^{2} f \cdot x^{2}+\cdots+\frac{1}{k !} d_{a}^{k} f \cdot x^{k}
$$

isto é, $j^{k} f(a)$ é a expansão em série de potências de $f(x+a)-f(a)$ em torno da origem truncada no termo de grau $k$.

\subsection{Espaço Tangente e Determinação finita}

Definição 1.2.1 Uma ação de um grupo $G$ em um conjunto $M$ é uma aplicação $\varphi: G \times M \rightarrow M$, denotada por $\varphi(g, x)=g . x$, tal que para todo $x \in M$ e $g, h \in G$ temos:

(i) $1 . x=x$, com 1 identidade de $G$;

(ii) $(g h) \cdot x=g \cdot(h \cdot x)$.

Dado $x \in M$, o conjunto $G . x=\{g . x \in M / g \in G\}$ é chamado órbita de $x$ em $M$. A relação dada por $y \sim x$ se $y \in G . x$ é uma relação de equivalência.

Definição 1.2.2 Um grupo de Lie é um grupo multiplicativo $G$ que é uma variedade diferenciável e as operações de multiplicação e inversão são diferenciáveis. Uma ação de grupo de Lie é uma ação de um grupo de Lie G em uma variedade diferenciável $M$ que seja diferenciável.

Em geral, as órbitas de uma ação de grupo de Lie numa variedade não são subvariedades e sim subvariedades imersas, mas nos casos em que G.x é variedade, vale o seguinte resultado:

Teorema 1.2.3 Seja $\varphi: G \times M \rightarrow M$ uma ação de grupo de Lie $G$ em uma variedade $M$. Se as órbitas são subvariedades de $M$, então para todo $x \in M$ a aplicação $\varphi_{x}: G \rightarrow G_{x}$ dada por $\varphi_{x}(g)=g . x$ é uma submersão. E mais, o espaço tangente à órbita $G . x$ em $x$ é a imagem de $d_{1} \varphi_{x}: T_{1} G \rightarrow T_{x} M$, isto é, $T_{x} G . x=d_{1} \varphi_{x}\left(T_{1} G\right)$.

Demonstração:Ver [12].

No que segue definiremos uma relação de equivalência entre $k$-jatos de maneira análoga à definida para germes.

Definição 1.2.4 Dois $k$-jatos $f, g \in J^{k}(n, p)$ são equivalentes se existem $\phi \in \operatorname{Diff}\left(\mathbb{C}^{n}\right) e$ $\psi \in \operatorname{Diff}\left(\mathbb{C}^{p}\right)$ tais que $j^{k}\left(\psi \circ f \circ \phi^{-1}\right)(0)=j^{k} g(0)$. 
A relação de equivalência definida acima provém de uma ação do grupo de Lie $\mathcal{A}^{k}$, que é o grupo dos $k$-jatos de elementos de $\mathcal{A}$, na variedade $J^{k}(n, p)$. O grupo $\mathcal{A}^{k}$ age em $J^{k}(n, p)$ da seguinte forma:

$$
\begin{aligned}
\varphi: \mathcal{A}^{k} \times J^{k}(n, p) & \longrightarrow J^{k}(n, p) \\
\left(j^{k} \phi(0), j^{k} \psi(0), f\right) & \longmapsto j^{k}\left(\psi \circ f \circ \phi^{-1}\right)(0)
\end{aligned}
$$

Definimos o grupo $\mathcal{A}_{1}$ como sendo o subgrupo de $\mathcal{A}$ cujos elementos têm o 1 -jato igual a identidade.

Proposição 1.2.5 O espaço tangente à órbita de $f \in J^{k}(n, p)$ segundo a ação do grupo $\mathcal{A}^{k}$, que denotamos por $T \mathcal{A}^{k} . f$, é

$$
T \mathcal{A}^{k} . f=\left\{j^{k}\left(\sum_{i=1}^{n} \frac{\partial f}{\partial x_{i}} g_{i}\right)(0) / g=\left(g_{1}, \ldots, g_{n}\right) \in J^{k}(n, n)\right\}+\left\{j^{k}(h \circ f)(0) / h \in J^{k}(p, p)\right\}
$$

Agora consideramos a órbita de um germe segundo a ação do grupo $\mathcal{A}$. Lembramos que este grupo não é de Lie e as órbitas não são subvariedades.

Definição 1.2.6 Seja $f \in \mathcal{O}_{n, p}$.

1. O espaço tangente à órbita de $f$ segundo a ação do grupo $\mathcal{A}$ é

$$
\text { TA.f }=\left\{\sum_{i=1}^{n} \frac{\partial f}{\partial x_{i}} g_{i} / g=\left(g_{1}, \ldots, g_{n}\right) \in \mathcal{M}_{n} \mathcal{O}_{n, n}\right\}+\left\{h \circ f / h \in \mathcal{M}_{p} \mathcal{O}_{p, p}\right\} .
$$

2. O espaço tangente estendido é

$$
T \mathcal{A}_{e} . f=\left\{\sum_{i=1}^{n} \frac{\partial f}{\partial x_{i}} g_{i} / g=\left(g_{1}, \ldots, g_{n}\right) \in \mathcal{O}_{n, n}\right\}+\left\{h \circ f / h \in \mathcal{O}_{p, p}\right\} .
$$

Definição 1.2.7 Definimos a $\mathcal{A}$-codimensão de $f$, que denotamos por $\mathcal{A}$-cod $f$, por

$$
\operatorname{dim}_{\mathbb{C}} \frac{\mathcal{M}_{n} \mathcal{O}_{n, p}}{T \mathcal{A} . f}
$$

e a $\mathcal{A}_{e}$-codimensão de $f$, que denotamos por $\mathcal{A}_{e}$-cod $f$, por

$$
\operatorname{dim}_{\mathbb{C}} \frac{\mathcal{O}_{n, p}}{T \mathcal{A}_{e} \cdot f}
$$

Dizemos que um germe $f \in \mathcal{O}_{n, p}$ é estável se $T \mathcal{A}_{e} . f=\mathcal{O}_{n, p}$. 
Definição 1.2.8 Um germe $f \in \mathcal{O}_{n, p}$ é $\mathcal{A}$ - $k$-determinado se para todo $g \in \mathcal{O}_{n, p}$ tal que $j^{k} g(0)=j^{k} f(0)$ temos que $g$ é $\mathcal{A}$-equivalente a $f$. Dizemos que um germe $f$ é $\mathcal{A}$-finitamente determinado se $f$ é $\mathcal{A}$ - $k$-determinado para algum $k$.

Teorema 1.2 .9 ( [31]) Seja $f \in \mathcal{O}_{n, p}$. São equivalentes:

(i) O germe fé $\mathcal{A}$-finitamente determinado,

(ii) Existe um inteiro positivo $k$ tal que $\mathcal{M}_{n}^{k} \mathcal{O}_{n, p} \subset T \mathcal{A} . f$,

(iii) $A \mathcal{A}$-codimensão de $f$ é finita,

(iv) $A \mathcal{A}_{e}$-codimensão de $f$ é finita.

O próximo resultado é uma caracterização geométrica dos germes finitamente determinados.

Teorema 1.2.10 Um germe $f \in \mathcal{M}_{n} \mathcal{O}_{n, p}$ é finitamente determinado se, e somente se, existe uma vizinhança $V$ da origem em $\mathbb{C}^{n}$ tal que para todo subconjunto finito $S \subset V-\{0\}, o$ multigerme de $f$ em $S$ é estável.

\subsection{Equivalência de contato}

Introduzimos nesta seção uma outra relação de equivalência entre germes $\mathbb{C}^{n}, x \rightarrow \mathbb{C}^{p}, y$. Esta relação é chamada de equivalência de contato ou $\mathcal{K}$-equivalência.

Definição 1.3.1 Dizemos que dois germes $f_{1}: \mathbb{C}^{n}, x_{1} \rightarrow \mathbb{C}^{p}, y_{1}$ e $f_{2}: \mathbb{C}^{n}, x_{2} \rightarrow \mathbb{C}^{p}, y_{2}$ são $\mathcal{K}$-equivalentes, e denotamos por $f_{1} \sim_{\mathcal{K}} f_{2}$, se existem germes de difeomorfismos $h: \mathbb{C}^{n}, x_{1} \rightarrow$ $\mathbb{C}^{n}, x_{2}$ e $H: \mathbb{C}^{n} \times \mathbb{C}^{p},\left(x_{1}, y_{1}\right) \rightarrow \mathbb{C}^{n} \times \mathbb{C}^{p},\left(x_{2}, y_{2}\right)$ para os quais o diagrama:

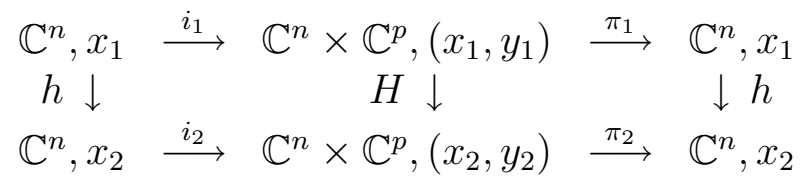

é comutativo, onde $i_{k}$ é o germe em $x_{k}$ da inclusão $\mathbb{C}^{n} \rightarrow \mathbb{C}^{n} \times \mathbb{C}^{p}$ dada por $x \mapsto\left(x, y_{k}\right)$ e $\pi_{k}$ é o germe em $\left(x_{k}, y_{k}\right)$ da projeção $\mathbb{C}^{n} \times \mathbb{C}^{p} \rightarrow \mathbb{C}^{n}$ dada por $(x, y) \mapsto x, k=1,2$. Em outras palavras, $H$ é dado por

$$
H(x, y)=(h(x), \theta(x, y))
$$

$\operatorname{com} \theta\left(x, y_{1}\right)=y_{2}$. Dizemos que $f_{1}$ e $f_{2}$ são $\mathcal{K}$-equivalentes se existem $h$ e $H$ como acima tais que

$$
H\left(1, f_{1}\right)=\left(1, f_{2}\right) \circ h
$$

Dizemos que um germe $f \in \mathcal{O}_{n, p}$ é $\mathcal{K}$-finitamente determinado se existe $l \in \mathbb{N}$ satisfazendo a seguinte condição: para todo $g \in \mathcal{O}_{n, p}$ tal que $j^{l} g(0)=j^{l} f(0)$ temos que $g$ é $\mathcal{K}$-equivalente a $f$.

Além disso, dois germes $\mathcal{A}$-equivalentes também são $\mathcal{K}$-equivalentes. 


\subsection{Desdobramentos}

Seja $f_{0} \in \mathcal{M}_{n} \mathcal{O}_{n, p}$. Um desdobramento a $s$-parâmetros de $f_{0}$ é um germe de uma aplicação

$$
\begin{aligned}
F: \mathbb{C}^{n} \times \mathbb{C}^{s}, 0 & \longrightarrow \mathbb{C}^{p} \times \mathbb{C}^{s}, 0 \\
(x, u) & \longmapsto(f(u, x), u)
\end{aligned}
$$

satisfazendo $f(0, x)=f_{0}(x)$. O germe $f: \mathbb{C}^{n} \times \mathbb{C}^{s}, 0 \rightarrow \mathbb{C}^{p}, 0$ é chamado deformação de $f_{0}$.

Definição 1.4.1 Dois desdobramentos $F, G: \mathbb{C}^{n} \times \mathbb{C}^{s}, 0 \longrightarrow \mathbb{C}^{p} \times \mathbb{C}^{s}, 0$ de $f_{0}$ são isomorfos se existem germes de difeomorfismos $\phi: \mathbb{C}^{n} \times \mathbb{C}^{s}, 0 \longrightarrow \mathbb{C}^{n} \times \mathbb{C}^{s}, 0$ e $\psi: \mathbb{C}^{p} \times \mathbb{C}^{s}, 0 \longrightarrow \mathbb{C}^{p} \times \mathbb{C}^{s}, 0$, desdobramentos a $s$-parâmetros dos germes das identidades em $\mathbb{C}^{n}$ e $\mathbb{C}^{p}$ respectivamente, tais que $G=\psi \circ F \circ \phi^{-1}$.

Seja $F$ um desdobramento a $s$-parâmetros de $f_{0} \in \mathcal{M}_{n} \mathcal{O}_{n, p}$ e $h: \mathbb{C}^{t}, 0 \rightarrow \mathbb{C}^{s}, 0$ um germe. Definimos $h^{*} F$ como o desdobramento a $t$-parâmetros de $f_{0}$ dado por

$$
\begin{aligned}
h^{*} F: \mathbb{C}^{n} \times \mathbb{C}^{t}, 0 & \longrightarrow \mathbb{C}^{p} \times \mathbb{C}^{t}, 0 \\
(x, v) & \longmapsto(f(h(v), x), v)
\end{aligned}
$$

Se $G$ é um desdobramento a $t$-parâmetros de $f_{0}$, dizemos que $G$ é induzido de $F$ se existe um germe $h: \mathbb{C}^{t}, 0 \rightarrow \mathbb{C}^{s}, 0$ tal que $G$ é isomorfo a $h^{*} F$. Em particular, se $h: \mathbb{C}^{s}, 0 \rightarrow \mathbb{C}^{s}, 0$ é um germe de difeomorfismo, dizemos que $F$ e $G$ são equivalentes.

Dizemos que $F$ é um desdobramento versal de $f_{0}$ se todos os desdobramentos de $f_{0}$ são induzidos de $F$. Quando $F$ é versal com um número mínimo de parâmetros dizemos que $F$ é miniversal.

O desdobramento $F$ é trivial se é isomorfo ao desdobramento constante $G: \mathbb{C}^{n} \times \mathbb{C}^{s}, 0 \longrightarrow$ $\mathbb{C}^{p} \times \mathbb{C}^{s}, 0, G(x, u)=\left(f_{0}(x), u\right)$.

Definição 1.4.2 Seja $f \in \mathcal{O}_{n, n}$ um germe $\mathcal{A}$-finitamente determinado. Então existe um desdobramento a um parâmetro de $f$

$$
\begin{aligned}
F: \mathbb{C}^{n} \times \mathbb{C}, 0 & \longrightarrow \mathbb{C}^{n} \times \mathbb{C}, 0 \\
(\mathbf{x}, t) & \longmapsto\left(f_{t}(\mathbf{x}, t), t\right)
\end{aligned}
$$

tal que $f_{t}$ é estável para $t$ suficientemente pequeno. A aplicação $f_{t}$ é chamada de perturbação estável de $f$. Assim, $\left\{f_{t}, t \in \mathbb{C}\right\}$ é uma família de germes em $\mathcal{M}_{n} \mathcal{O}_{n, n}$ com $f_{0}=f$ e $f_{t}$ estável para todo $t \neq 0$. 
Definição 1.4.3 Dizemos que um germe $f \in \mathcal{M}_{n} \mathcal{O}_{n, p}$ é $\mathcal{A}$-simples se existe um número finito de classes de equivalência tal que se $f$ é mergulhado em qualquer família $F: \mathbb{C}^{n} \times P,\left(0, p_{0}\right) \rightarrow \mathbb{C}^{p}, 0$, então para todo $(x, p)$ numa vizinhança suficientemente pequena de $\left(0, p_{0}\right)$, o germe de $f_{p}=F(x, p)$ em $x$ está em uma dessas classes de equivalência. 


\section{Capítulo 2}

\section{Invariantes}

\subsection{Preliminares}

Seja $f: \mathbb{C}^{n}, 0 \rightarrow \mathbb{C}^{n}, 0$ um germe de aplicação de coposto 1 dado pela pré-forma normal

$$
(\mathbf{x}, y) \mapsto(\mathbf{x}, g(\mathbf{x}, y))
$$

$(\mathbf{x}, y) \in \mathbb{C}^{n-1} \times \mathbb{C}$ e seja

$$
\tilde{f}=\tilde{f}_{1}, \ldots, \tilde{f}_{s}: \mathbb{C}^{n}, S \rightarrow \mathbb{C}^{n}, q
$$

onde $S=\left\{p_{1}, \ldots, p_{s}\right\}, \tilde{f}(S)=\{q\}$ e $\tilde{f}_{i}(\mathbf{x}, y)=\left(\mathbf{x}, \tilde{g}_{i}(\mathbf{x}, y)\right), i=1, \ldots, s$, um $s$-germe aparecendo numa deformação de $f$.

As $\mathcal{K}$-classes de germes equidimensionais de coposto 1 são as do tipo $A_{k}$, com representantes $\left(\mathbf{x}, y^{k+1}\right)$, e as $\mathcal{K}$-classes de $s$ - germes são do tipo $A_{\left(k_{1}, \ldots, k_{s}\right)}$, onde os $s$ pontos $p_{i}, i=1, \ldots, s$, da fonte são singularidades do tipo $A_{k_{i}}, i=1, \ldots, s$, de $\tilde{f}_{i}$ e têm a mesma imagem.

Os multigermes equidimensionais estáveis de coposto 1 são aqueles que são transversais às suas $\mathcal{K}$-classes $A_{\left(k_{1}, \ldots, k_{s}\right)}$ e as singularidades estáveis isoladas são, entre essas, aquelas em que $\sum_{i=1}^{s} k_{i}=n$, ver [12, pag. 206].

Para relações envolvendo multigermes também consideramos $(s+l)$-germes compostos por $s$-germes do tipo $A_{\left(k_{1}, \ldots, k_{s}\right)}$ e $l$ pontos regulares $p_{s+1}, \ldots, p_{s+l}$ ( e todos os $p_{i}$ são levados no mesmo ponto da meta), denotamos esses $(s+l)$-germes por $A_{\left(k_{1}, \ldots, k_{s}, 0_{l}\right)}$.

Para germes $\mathcal{A}$-finitamente determinados os fechos do conjuntos de germes do tipo $A_{\left(k_{1}, \ldots, k_{s}, 0_{l}\right)}$ são interseções completas em $\left(\mathbb{C}^{n}\right)^{s+l}$ de codimensão $n-\sum_{i=1}^{s} k_{i}$. Para $l=0$ as equações que definem $\bar{A}_{\left(k_{1}, \ldots, k_{s}, 0_{l}\right)}$ foram descritas em [26] e em [14]. No que segue vamos descrevê-las e estendê-las ao caso $l>0$.

Para $(s+l)$-germes de coposto 1 podemos identificar $\left(\mathbb{C}^{n}\right)^{s+l}$ com $\mathbb{C}^{n+s+l-1}$, com coordenadas $\left(\mathbf{x}, y_{1}, \ldots, y_{s+l}\right)$. E para este último usaremos coordenadas $\left(\mathbf{x}, y_{1}, \epsilon_{2}, \ldots, \epsilon_{s+l}\right)$, onde 
$e_{i}=y_{i+1}-y_{i}, i=2, \ldots, s+l$. Um ponto $\left(\mathbf{x}, y, \epsilon_{2}, \ldots, \epsilon_{s+l}\right) \in \mathbb{C}^{n+s+l-1}$ corresponde a:

$$
\left(\left(\mathbf{x}, y_{1}\right),\left(\mathbf{x}, y_{1}+\epsilon_{2}\right), \ldots,\left(\mathbf{x}, y_{1}+\sum_{i=2}^{s+l} \epsilon_{i}\right)\right) \in\left(\mathbb{C}^{n}\right)^{s+l} \text {. }
$$

Seja $k(s, m)=\left(k_{1}, \ldots, k_{s}\right)$ uma partição de $m$ de comprimento $s$. Denotamos por $\left(k_{1}, \ldots, k_{s}, 0_{l}\right)=k(s, m), 0_{l}$, a partição $k(s, m)$ aumentada por uma sequência de zeros de comprimento $l$. Quando $s, m$ e $l$ são claramente identificados no contexto, vamos denotar $k(s, m), 0_{l}$ por $\sigma$.

Os conjuntos $\bar{A}_{k(s, m), 0_{l}} \subset \mathbb{C}^{n+s+l-1}$ são dados pelos zeros das aplicações

$$
G_{k(s, m), 0_{l}}=\left(G_{1}, \ldots, G_{m+s+l-1}\right): \mathbb{C}^{n+s+l-1} \rightarrow \mathbb{C}^{m+s+l-1},
$$

cujas funções componentes $G_{i}$ são definidas como segue. Definimos e $g_{1}^{(i)}=\partial^{i} g / \partial y_{1}^{i}, i \geq 1$ e para $j=1, \ldots, s+l-1$

$$
g_{j+1}^{(0)}:=\sum_{\alpha \geq k_{j}+1} g_{j}^{(\alpha)} \epsilon_{j+1}^{\alpha-k_{j}-1} / \alpha !, \quad g_{j+1}^{(i)}:=\partial^{i} g_{j+1}^{(0)} / \partial \epsilon_{j+1}^{i}, \quad i \geq 1
$$

Então as funções componentes de $G_{k(s, m), 0_{l}}$ são, nesta ordem, $g_{1}^{(i)}\left(i=1, \ldots, k_{1}\right)$ e $g_{j}^{(i)}$ $\left(i=0, \ldots, k_{j}, j=2, \ldots, s+l\right)$. Observamos que $k_{j} \geq 1$ para $j=2, \ldots, s$ e $k_{j}=0$ para $j>s$.

Exemplo 2.1.1 Para um germe $\mathcal{K}$-finitamente determinado de multiplicidade local $m_{f}(0)=d$ com pré-forma normal $f=\left(\mathbf{x}, y_{1}^{d}+P_{1}(\mathbf{x}) y_{1}+\ldots+P_{d-2}(\mathbf{x}) y_{1}^{d-2}\right)$, obtemos para $j=1, \ldots, s+l-1$

$$
g_{j+1}^{(0)}=\epsilon_{j+1}^{d-k_{1}-\ldots-k_{j}-j}+Q\left(\mathbf{x}, y_{1}, \epsilon_{2}, \ldots, \epsilon_{j}\right) \epsilon_{j+1}^{d-k_{1}-\ldots-k_{j}-j-1}+\ldots,
$$

ou seja, polinômios mônicos em $\epsilon_{j+1}$ de grau $d-k_{1}-\ldots-k_{j}-j$ cujos coeficientes são funções suaves em $\left(\mathbf{x}, y_{1}, \epsilon_{2}, \ldots, \epsilon_{j}\right)$.

O seguinte resultado está em [26]:

Lema 2.1.2 Dado um par de germes de coposto 1 equidimensionais $f$ e $f^{\prime}$ os correspondentes pares de germes $G_{k(s, m)}$ e $G_{k(s, m)}^{\prime}$ são $\mathcal{K}$-equivalentes.

Ou seja, $\mathcal{K}$-invariantes de $G_{k(s, m)}$ são $\mathcal{A}$-invariantes de $f$ e o mesmo é válido para as aplicações $G_{k(s, m), 0_{l}}$ (pelo mesmo argumento). Sejam $\mu_{\sigma}(f)$ e $\tau_{\sigma}(f)$ os números de Milnor e de Tjurina de $G_{\sigma}$, respectivamente.

Definição 2.1.3 $\quad$ (i) Sejam $\mathcal{M}_{m+s+l-1}$ o ideal maximal de $\mathcal{O}_{m+s+l-1}$ e $\overline{A_{\sigma}}$ o conjunto dos zeros do ideal $G_{\sigma}^{*} \mathcal{M}_{m+s+l-1}$. Definimos $\mathcal{I}\left(A_{\sigma}\right)=G_{\sigma}^{*} \mathcal{M}_{m+s+l-1}$.

(ii) Seja $J\left(G_{\sigma}\right)$ o ideal gerado pelos determinantes das maiores submatrizes de $d G_{\sigma}(\mathbf{x}, y, \epsilon)$ então

$$
v_{\sigma}(f)=\operatorname{dim}_{\mathbb{C}} \frac{\mathcal{O}_{n+s+l-1}}{\mathcal{I}\left(A_{\sigma}\right)+J\left(G_{\sigma}\right)}
$$


Teorema 2.1.4 ( [31],Teorema 4.5.1) (i) Para um germe quase-homogêneo $f$ e $m<n$, temos que

$$
\mu_{\sigma}(f)=v_{\sigma}(f)
$$

(ii) Para $m=n$ temos que

$$
v_{\sigma}(f)=\mu_{\sigma}(f)=m_{G_{\sigma}}(0)-1
$$

Definição 2.1.5 O número de $A_{\sigma}$-pontos isolados aparecendo em uma perturbação estável de $f$ é dado por

$$
r_{\sigma}(f)=c^{-1} \cdot m_{G_{\sigma}}(0)=c^{-1}\left(v_{\sigma}+1\right)=c^{-1}\left(\mu_{\sigma}+1\right),
$$

onde c denota o número de permutações dos $s+l$ pontos na fonte que preservam $\sigma$. Então, para

$$
\sigma=(\underbrace{k_{1}, \ldots, k_{1}}_{b_{1} \text { vezes }}, \ldots, \underbrace{k_{r}, \ldots, k_{r}}_{b_{k} \text { vezes }}, 0_{l}), b_{1} k_{1}+\ldots+b_{r} k_{r}+=n, \sum_{i=1}^{k} b_{i}=s
$$

temos $c=l ! \prod_{i=1}^{r}\left(b_{i} !\right)$.

Este fator $c$ é igual ao grau da aplicação $f^{s+l}: \bar{A}_{\sigma} \rightarrow \mathbb{C}^{n}$, que é dado pela restrição da aplicação $\mathbb{C}^{n+s+l-1} \rightarrow\left(\mathbb{C}^{n}\right)^{l+s}$, definida por

$$
\left(\mathbf{x}, y, \epsilon_{2}, \ldots, \epsilon_{s+l}\right) \mapsto\left(f(\mathbf{x}, y), f\left(\mathbf{x}, y+\epsilon_{2}\right), \ldots, f\left(\mathbf{x}, y+\sum_{i=2}^{s+l} \epsilon_{i}\right)\right),
$$

à interseção da diagonal principal na meta com o extrato $A_{\sigma}$ do discriminante de $f$.

Teorema 2.1.6 ( [26],Teorema 2.6) Seja $f: \mathbb{C}^{n}, 0 \rightarrow \mathbb{C}^{n}, 0$, um germe de aplicação de coposto 1.

(i) São equivalentes:

(a) $f$ é $\mathcal{A}$-finitamente determinado.

(b) $v_{k(s, m)}(f)<\infty$ para todas as partições $k(s, m), 2 \leq m \leq n$ e $m+s \leq m_{f}(0)$.

(c) $v_{k(s, m)}(f)<\infty$ para todas as partições de $m=2, \ldots, n$ com parcelas $k_{i} \in\{1,2\}$ e $m+s \leq m_{f}(0)$.

(ii) Os números $v_{k(s, m)}(f)$ são $\mathcal{A}$-invariantes. 
Concluímos este capítulo com algumas observações sobre os invariantes definidos acima: em 2.1.1 descrevemos algumas relações entre os invariantes e a $\mathcal{A}$-finitude de $f$. Observamos que os números de Milnor dos conjuntos $A_{\sigma}$ são $\mathcal{A}$-invariantes topológicos de $f$, e no Lema 2.1.8 descrevemos algumas propriedades algébricas desses números de Milnor (um tipo de aditividade de codimensões). E 2.1.12 contém uma observação sobre a semi-quasi-homogeneidade de $f$ e as aplicações associadas $G_{\sigma}$.

Observações 2.1.1 São equivalentes:

1. $A_{\sigma}$ é uma ICIS.

2. A aplicação $G_{\sigma}$ é $\mathcal{K}$-finitamente determinada.

3. $v_{\sigma}(f)<\infty$ (de fato, $v_{\sigma}(f)$ é a codimensão do ideal cujos zeros estão no conjunto dos $\mathcal{K}$ pontos instáveis de $G_{\sigma}$ na fonte, e a codimensão é finita se, e somente se, 0 é um $\mathcal{K}$-ponto instável).

4. $\mu_{\sigma}(f)<\infty$.

Segue do Teorema acima que: $f$ é $\mathcal{A}$-finitamente determinado se, e somente se, as aplicações associadas $G_{\sigma}$ são $\mathcal{K}$-finitamente determinadas para toda partição $\sigma=\left(k_{1}, \ldots, k_{s}\right)$ de $m=2, \ldots, n$ com parcelas $k_{i} \in\{1,2\}$.

Agora vamos discutir uma propriedade algébrica dos invariantes $\mu_{\sigma}(f)$ e $v_{\sigma}(f)$. Seja $R=\mathcal{O}_{n+s+l-1}$ e $\operatorname{col}(\mathcal{I})=\operatorname{dim}_{\mathbb{C}} R / \mathcal{I}$ denota a codimensão de um ideal $\mathcal{I}$ no anel local $R$. Sejam $h_{0}$ a última função componente da aplicação $G_{\sigma}, z$ a última coordenada em $\mathbb{C}^{n+s+l-1}$ (que é dada por $y$ ou $\epsilon_{s+l}$ ) e $h=\partial h_{0} / \partial z$. Além disso, seja $A:=\mathcal{I}\left(A_{\sigma}\right)$, e $B$ e $C$ denotam os ideais gerados pelos determinantes das maiores submatrizes de $d G_{\sigma}$ sem a última coluna (para $B$ ) e sem a última linha e a última coluna (para $C$ ). Quando o número de linhas excede o número de colunas de $d G_{\sigma}$, consideraremos $B$ e $C$ como ideais nulos. Então

$$
v_{\sigma}(f)=\operatorname{col}\left(A+J\left(G_{\sigma}\right)\right)=\operatorname{col}(A+B+h C) .
$$

O próximo Teorema é de grande importância para demonstrar o principal resultado deste trabalho:

\section{Teorema 2.1.7 (Lê [19], Greuel [15])}

Seja $X$ uma ICIS com singularidade na origem, $X^{\prime}$ uma ICIS definida em $X$ por $f_{k}=0$, e sejam $f_{1}, \ldots, f_{k-1}$ os geradores do ideal que define $X$. Então:

$$
\mu(X, 0)+\mu\left(X^{\prime}, 0\right)=\operatorname{dim}_{\mathbb{C}} \frac{\mathcal{O}_{n}}{\left\langle f_{1}, \ldots, f_{k-1}, J\left(f_{1}, \ldots, f_{k}\right)\right\rangle}
$$


O teorema acima é, na verdade, somente um lema usado para calcular números de Milnor de ICIS. E uma boa referência para tal também é encontrada em Looijenga [20].

Para os números de Milnor $\mu_{\sigma}(f)=\mu\left(G_{\sigma}\right)$ e $\mu_{\sigma}\left(f^{\prime}\right)=\mu\left(\left(x_{1}, G_{\sigma}\right)\right)$ temos, pela fórmula de Lê e Greuel que

$$
\mu_{\sigma}(f)+\mu_{\sigma}\left(f^{\prime}\right)=\operatorname{col}\left(A+J\left(G_{\sigma}^{\prime}\right)\right)=\operatorname{col}\left(A+B^{\prime}+h C^{\prime}\right),
$$

onde $h$ e $A$ são como acima e $B^{\prime}$ e $C^{\prime}$ denotam os ideais gerados pelos determinantes das maiores submatrizes de $d G_{\sigma}$ sem a primeira e a última coluna (para $B^{\prime}$ ) e sem a primeira coluna, a última linha e a última coluna (para $\left.C^{\prime}\right)$.

Lema 2.1.8 Sejam $R$ um domínio, $a \in R$ e $I, J$ e $J^{\prime}$ ideais em $R$, com $J \subset J^{\prime}$. Então existe um inteiro não negativo $\delta$ tal que

$$
\operatorname{col}\left(I+J+a J^{\prime}\right)=\operatorname{col}(I+J+(a))+\operatorname{col}\left(I+J^{\prime}\right)-\delta .
$$

\section{Demonstração:}

Consideramos a sequência exata:

$$
0 \longrightarrow R /(I+J): a+J^{\prime} \longrightarrow R / I+J+a J^{\prime} \longrightarrow R / I+J+(a) \longrightarrow 0 .
$$

A terceira aplicação é o epimorfismo cujo núcleo é gerado pelas classes de $a$ módulo $I+J+$ $a J^{\prime}$. Este núcleo é isomorfo a $(a) /(a) \cap\left(I+J+a J^{\prime}\right)$, que é igual a

$$
(a) /(I+J) \cap(a)+a J^{\prime} \cong(a) / a\left((I+J): a+J^{\prime}\right)
$$

(usando a lei modular e a fórmula $a(\mathcal{I}: a)=\mathcal{I} \cap(a)$ ). A aplicação

$$
R /(I+J): a+J^{\prime} \rightarrow(a) / a\left((I+J): a+J^{\prime}\right),
$$

dada por multiplicação por $a$, é um isomorfismo. Então:

$$
\operatorname{col}\left(I+J+a J^{\prime}\right)=\operatorname{col}(I+J+(a))+\operatorname{col}\left((I+J): a+J^{\prime}\right)
$$

Como $I+J^{\prime} \subset(I+J): a+J^{\prime}$ segue que existe $\delta \in \mathbb{N}$ tal que

$$
\operatorname{col}\left(I+J+a J^{\prime}\right)=\operatorname{col}(I+J+(a))+\operatorname{col}\left(I+J^{\prime}\right)-\delta .
$$

Corolário 2.1.9 Tomando $I=A, J=B^{\prime}, J^{\prime}=C^{\prime}$ e $a=h$, temos

$$
\mu_{\sigma}(f)+\mu_{\sigma}\left(f^{\prime}\right)=\operatorname{col}\left(A+B^{\prime}+h C^{\prime}\right)=\operatorname{col}\left(A+B^{\prime}+(h)\right)+\operatorname{col}\left(A+C^{\prime}\right),
$$

isto é, $\delta=0$ 


\section{Demonstração:}

Lembramos o seguinte fato sobre ideais determinantais em um anel Cohen-Macaulay $R$ (veja, por exemplo, [5]). Seja $\mathcal{I}_{r}$ o ideal gerado pelos maiores menores de uma matriz $r$ por $t(r \leq t)$ com entradas em $R$. O ideal $\mathcal{I}_{r}$ tem codimensão no máximo $t-r+1$, e $R / \mathcal{I}_{r}$ é Cohen-Macaulay se $\mathcal{I}_{r}$ tem codimensão exatamente $t-r+1$. No caso $\mu_{\sigma}$ as dimensões de $R / A+B, R / A+B+h C$ e $R / A+B+(h)$ são pelo menos 1,0 e 0 , e da finitude de $\mu_{\sigma}(f)+\mu_{\sigma}\left(f^{\prime}\right)$ vemos que essas dimensões são de fato iguais a esses números.

Neste caso, $R / A+B^{\prime}$ é Cohen-Macaulay de dimensão 1 e $A+B^{\prime}+(h)$ tem comprimento finito, logo $h$ não é um divisor de zero em $A+B^{\prime}$,ou seja, não é um divisor de zero em $\left(A+B^{\prime}\right): h=A+B^{\prime}$. Mas $A+B^{\prime}+C^{\prime}=A+C^{\prime}$, portanto $\delta=0$.

Observação 2.1.10 No caso $v_{\sigma}$ temos (para $v_{\sigma}(f)<\infty$ ) que $A+B$ tem comprimento finito, e o comprimento de $A+B+(h)$ é em geral não nulo. Portanto $(A+B): h \neq A+B$, em geral. Em muitos casos temos $(A+B): h+C=A+C$, tal que $\delta_{\sigma}=0$, mas aqui segue um exemplo simples onde isto não ocorre. Considere $v_{(2)}(f)$ para $f: \mathbb{C}^{2}, 0 \rightarrow \mathbb{C}^{2}, 0$ dado por $f=\left(x, y^{4}+x^{2} y\right)$. Então $A=\left(4 y^{3}+x^{2}, y^{2}\right), B=0, C=(x)$ e $h=(y)$. Portanto $\operatorname{col}(A+B+h C)=3$, mas $\operatorname{col}(A+B+(h))=2=\operatorname{col}(A+C)$, e $\delta_{(2)}=\operatorname{col}(A+C)-\operatorname{col}((A+B): h+C)=1$.

Definição 2.1.11 Um germe $f$ é semi-quase-homogêneo (em relação à $\mathcal{A}$ - equivalência) se $f=f_{0}+f_{+}$, onde a parte inicial $f_{0}$ é quase homogênea e $\mathcal{A}$-finitamente determinada e $f_{+}$tem grau pesado mais alto do que $f_{0}$.

Observação 2.1.12 As aplicações associadas $G_{k(s, m), 0_{l}}$ então serão semi-quase homogêneas em relação à $\mathcal{K}$-equivalência (com os pesos de $x_{i}$ e $y$ iguais para $f_{0}$ e grau $\left(\epsilon_{i}\right)=\operatorname{grau}(y)$, $j=2, \ldots, s+l)$ - observamos que as partes iniciais das aplicações $G_{k(s, m), 0_{l}}$, correspondentes a $f_{0}$, são $\mathcal{K}$-finitamente determinadas, porque $f_{0}$ é $\mathcal{A}$-finitamente determinada. Para $f$ com parte inicial não $\mathcal{A}$-finitamente determinada, as partes iniciais de $G_{k(s, m), 0_{l}}$ ainda podem ser $\mathcal{K}$-finitamente determinadas. 


\section{Capítulo 3}

\section{Relações entre invariantes de germes de coposto 1 de $\mathbb{C}^{n}$ em $\mathbb{C}^{n}$}

Lembramos que as relações aqui apresentadas generalizam os resultados de Gaffney e Mond para o números de cúspides e o número de pontos duplos presentes numa deformação estável de germes de $\mathbb{C}^{2}$ em $\mathbb{C}^{2}$, ver [11].

\subsection{Relações entre invariantes}

Começamos com o seguinte resultado:

Proposição 3.1.1 (Proposição 5.12, [20]) Seja $f: \mathbb{C}^{n}, 0 \rightarrow \mathbb{C}^{n}, 0$, um germe de aplicação de coposto 1, que define uma ICIS X com singularidade na origem. Então

$$
\mu(X, 0)=m_{f}(0)-1,
$$

onde $m_{f}(0)=\operatorname{dim}_{\mathbb{C}} \frac{\mathcal{O}_{n}}{\left(f_{1}, \ldots, f_{n}\right)}$.

Como o germe $f$ tem coposto $1, f(\mathbf{x}, y) \mapsto(\mathbf{x}, g(\mathbf{x}, y))$, sua multiplicidade se reduz ao menor expoente de um monômio puro em $y$, encontrado em $g(\mathbf{x}, y)$.

As fórmulas a seguir relacionam números de Milnor de certas aplicações e a multiplicidade local de um germe de coposto 1. Este teorema é o principal resultado do nosso trabalho e usam fortemente as fórmulas obtidas por Lê e Greuel.

Teorema 3.1.2 ( [3]) Seja $f: \mathbb{C}^{n}, 0 \rightarrow \mathbb{C}^{n}, 0, n \geq 1$, um germe de aplicação de coposto 1 e multiplicidade local $m_{f}(0)=r$, seja $k(s, n)$ uma partição de $n$ como anteriormente. Suponhamos que $\mu_{(k(s, n))}<\infty$, ou seja, todos os invariantes que aparecem nas fórmulas são finitos. Então

(i) Se $s=1$ e $r \geq n+1$ temos:

$$
\mu_{(n)}(f)-\mu_{(n-1)}(f)+\ldots+(-1)^{n+1} \mu_{(1)}(f)=m_{f}(0)-n-1 . \quad\left[F_{n}(r)\right]
$$


(ii) Se $s=1$ e $r \leq n$ temos:

$$
\mu_{(r-1)}(f)-\mu_{(r-2)}(f)+\ldots+(-1)^{r} \mu_{(1)}(f)=0 . \quad\left[F_{n}(r)\right]
$$

(iii) Se $s>1, k_{1}+\ldots+k_{s}=n, k_{i} \geq 1$ (para $i<s$ ) e $k_{s} \geq 0$, e $r \geq n+s$ temos:

$$
\begin{gathered}
\mu_{\left(k_{1}, \ldots, k_{s}\right)}(f)-\mu_{\left(k_{1}, \ldots, k_{s}-1\right)}(f)+\ldots+(-1)^{k_{s}} \mu_{\left(k_{1}, \ldots, k_{s-1}, 0\right)}(f) \\
+(-1)^{k_{s}+1}\left(m_{f}(0)-\sum_{i=1}^{s-1}\left(k_{i}+1\right)\right) \mu_{\left(k_{1}, \ldots, k_{s-1}\right)}(f)=m_{f}(0)-n-s \quad\left[F_{\left(k_{1}, \ldots, k_{s}\right)}(r)\right]
\end{gathered}
$$

(iv) Suponhamos $r<n+s$. Seja

$$
t= \begin{cases}r-\left(k_{1}+\ldots+k_{s-1}+s\right) & \text { se } r>k_{1}+\ldots+k_{s-1}+s \\ 0 & \text { caso contrário }\end{cases}
$$

então

$$
\begin{gathered}
\mu_{\left(k_{1}, \ldots, k_{s-1}, t\right)}(f)-\mu_{\left(k_{1}, \ldots, k_{s-1}, t-1\right)}(f)+\ldots+(-1)^{t} \mu_{\left(k_{1}, \ldots, k_{s-1}, 0\right)}(f) \\
+(-1)^{t+1}(t+1) \mu_{\left(k_{1}, \ldots, k_{s-1}\right)}(f)=0 \quad\left[F_{\left(k_{1}, \ldots, k_{s}\right)}(r)\right]
\end{gathered}
$$

\section{Demonstração:}

(i) A demonstração é por indução sobre $n$. Para $n=1$ a afirmação segue da Proposição 3.1.1. portanto consideremos $n \geq 2$ e suponhamos que $F_{(n-1)}(r)$ vale. A condição de que todos os invariantes presentes em $F_{(n)}(r)$ são finitos nos garante que $r<\infty$ e que cada conjunto $\bar{A}_{(i)}$ não vazio é uma interseção completa com um ponto singular isolado na origem (no mínimo). Escolhendo coordenadas $\left(x_{1}, \ldots, x_{n-1}, y\right)$ na fonte tal que $f=\left(x_{1}, \ldots, x_{n-1}, g\left(x_{1}, \ldots, x_{n-1}, y\right)\right)$, onde $g(\mathbf{x}, y)=y^{r}+P_{1}(x) y+\ldots+P_{r-2}(x) y^{r-2}$, $P_{i} \in \mathcal{M}_{n-1}$, e $x_{1}=0$ é um hiperplano genérico para a sequência encaixante de subconjuntos

$$
\bar{A}_{(n-1)} \subset \bar{A}_{(n-2)} \subset \ldots \subset \bar{A}_{(1)}=\Sigma_{f} \subset \mathbb{C}^{n}, 0 .
$$

(Note que a propriedade " $\bar{A}_{(i)}$ é uma ICIS implica $\bar{A}_{(i)} \cap\left\{x_{1}=0\right\}$ é uma ICIS", $i=1, \ldots, n-1$, é suficientemente "genérica"). Segue da hipótese de indução que a fórmula $F_{(n-1)}(r)$ vale para $f^{\prime}:=\left.f\right|_{\left\{x_{1}=0\right\}}$, pois os invariantes de $f^{\prime}$ em $F_{(n-1)}(r)$ são finitos. Sejam $G_{(i)}$ e $G_{(i)}^{\prime}$ as aplicações como em 2.1 relativas a $f$ e $f^{\prime}$, respectivamente, consideremos $\mu_{(i)}:=\mu_{(i)}(f)=\mu\left(G_{(i)}\right)$ e $\mu_{(i)}^{\prime}:=\mu_{(i)}\left(f^{\prime}\right)=\mu\left(G_{(i)}^{\prime}\right)=\mu\left(x_{1}, G_{(i)}\right)$. 
Sejam $\mathcal{I}\left(M_{(i)}\right)$ e $\mathcal{I}\left(\tilde{M}_{(i)}\right)$ os ideais gerados pelos determinantes das maiores submatrizes do Jacobiano de $G_{(i)}$, omitindo a primeira e a última coluna de $d G_{(i)}$ no caso $\mathcal{I}\left(M_{(i)}\right)$ e somente a primeira coluna no caso $\mathcal{I}\left(\tilde{M}_{(i)}\right)$ (observamos que a primeira e a última coluna correspondem a $\partial / \partial x_{1}$ e $\partial / \partial y$, respectivamente).

Seja $\mathcal{I}\left(A_{(i)}\right)$ o ideal em $\mathcal{O}_{n}$ definido pelas funções componentes da aplicação $G_{(i)}$. Se $\operatorname{col}(\mathcal{I})$ denota o comprimento do ideal $\mathcal{I}$ em $\mathcal{O}_{n}$, ou seja, $\operatorname{col}(\mathcal{I})=\operatorname{dim}_{\mathbb{C}} \mathcal{O}_{n} / \mathcal{I}$. Segue de 2.1 .7 que

$$
\mu_{(i)}+\mu_{(i)}^{\prime}=\operatorname{col}\left(\mathcal{I}\left(A_{(i)}\right)+\mathcal{I}\left(\tilde{M}_{(i)}\right)\right)
$$

para $i=1, \ldots, n-1$. A soma dos números de Milnor $\mu_{(i)}+\mu_{(i)}^{\prime}$ no lado esquerdo dessa fórmula é finito, portanto o comprimento no lado direito da fórmula também é finito. Para $i=2, \ldots, n-2$ isto implica que

$$
\mu_{(i)}+\mu_{(i)}^{\prime}=\operatorname{col}\left(\mathcal{I}\left(A_{(i+1)}\right)+\mathcal{I}\left(M_{(i)}\right)\right)+\operatorname{col}\left(\mathcal{I}\left(A_{(i)}\right)+\mathcal{I}\left(M_{(i-1)}\right)\right) .
$$

Observamos que, módulo $\mathcal{I}\left(A_{(i)}\right)$, podemos escrever $\mathcal{I}\left(\tilde{M}_{(i)}\right)=g_{i+1} \mathcal{I}\left(M_{(i-1)}\right)+\mathcal{I}\left(M_{(i)}\right)$. Podemos então aplicar o Lema 2.1.8 para $R=\mathcal{O}_{n}, A=\mathcal{I}\left(A_{(i)}\right), B=\mathcal{I}\left(M_{(i)}\right)$, $C=\mathcal{I}\left(M_{(i-1)}\right)$ e $h=g_{i+1}$.

Para $i=n-1$ temos $\mathcal{I}\left(\tilde{M}_{(n-1)}\right)=g_{n} \mathcal{I}\left(M_{(n-2)}\right)$ módulo $\mathcal{I}\left(A_{(n-1)}\right)$, portanto

$$
\mu_{(n-1)}+\mu_{(n-1)}^{\prime}=\mu_{(n)}+1+\operatorname{col}\left(\mathcal{I}\left(A_{(n-1)}\right)+\mathcal{I}\left(M_{(n-2)}\right)\right) .
$$

(Aqui $\operatorname{col}\left(\mathcal{I}\left(A_{(n)}\right)\right)=\mu_{(n)}+1$ e, na notação do Lema 2.1.8, $J$ é o ideal nulo.)

Para $i=1$ temos

$$
\mu_{(1)}+\mu_{(1)}^{\prime}=\operatorname{col}\left(\mathcal{I}\left(A_{(2)}\right)+\mathcal{I}\left(M_{(1)}\right)\right) .
$$

Considerando as somas alternadas de (1), (2) e (3) para $i=n-1, n-2, \ldots, 1$ e aplicando $F_{(n-1)}(r)$ para $f^{\prime}$ obtemos a fórmula $F_{(n)}(r)$.

Com o intuito de facilitar o entendimento faremos o caso $n=3$. Devemos mostrar então que:

$$
\mu_{(3)}(f)-\mu_{(2)}(f)+\mu_{(1)}(f)=m_{f}(0)-3-1 . \quad\left[F_{3}(r)\right]
$$

Escolhendo coordenadas $\left(x_{1}, x_{2}, y\right)$ na fonte tal que $f=\left(x_{1}, x_{2}, g\left(x_{1}, x_{2}, y\right)\right)$, então $f^{\prime}=\left.f\right|_{\left\{x_{1}=0\right\}}\left(x_{2}, y\right)=\left(x_{2}, g\left(0, x_{2}, y\right)\right)$. Observe que as multiplicidades locais de $f \mathrm{e}$ $f^{\prime}$ são iguais. Por hipótese de indução o Teorema vale para $f^{\prime}$ e temos:

$$
\mu_{(2)}\left(f^{\prime}\right)-\mu_{(1)}\left(f^{\prime}\right)=m_{f^{\prime}}(0)-2-1=m_{f}(0)-3 . \quad\left[F_{2}(r)\right]
$$

onde $\mu_{(2)}\left(f^{\prime}\right)=\mu\left(x_{1}, G_{(2)}\right)=\mu\left(x_{1}, g_{y}, g_{y y}\right)$ e $\mu_{(1)}\left(f^{\prime}\right)=\mu\left(x_{1}, G_{(1)}\right)=\mu\left(x_{1}, g_{y}\right)$. Segue do Teorema 2.1.7 que 


$$
\mu_{(2)}(f)+\mu_{(2)}\left(f^{\prime}\right)=\mu\left(G_{(2)}\right)+\mu\left(x_{1}, G_{(2)}\right)=\operatorname{col}\left(G_{(2)}, J\left(x_{1}, G_{(2)}\right)\right)
$$

onde

$$
J\left(x_{1}, G_{(2)}\right)=\left|\begin{array}{ccc}
1 & 0 & 0 \\
g_{y x_{1}} & g_{y x_{2}} & g_{y y} \\
g_{y y x_{1}} & g_{y y x_{2}} & g_{y y y}
\end{array}\right|=g_{y x_{2}} g_{y y y}-g_{y y} g_{y y x_{2}}
$$

Então

$$
\begin{gathered}
\mu_{(2)}(f)+\mu_{(2)}\left(f^{\prime}\right)=\operatorname{col}\left(g_{y}, g_{y y}, g_{y x_{2}} g_{y y y}-g_{y y} g_{y y x_{2}}\right)=\operatorname{col}\left(g_{y}, g_{y y}, g_{y x_{2}} g_{y y y}\right)= \\
=\operatorname{col}\left(G_{(3)}\right)+\operatorname{col}\left(G_{(2)}, g_{y x_{2}}\right)=\mu_{(3)}(f)+1+\operatorname{col}\left(G_{(2)}, g_{y x_{2}}\right) \quad[(1)]
\end{gathered}
$$

Segue do Teorema 2.1.7 que:

$$
\mu_{(1)}(f)+\mu_{(1)}\left(f^{\prime}\right)=\mu\left(G_{(1)}\right)+\mu\left(x_{1}, G_{(1)}\right)=\operatorname{col}\left(G_{(1)}, J\left(x_{1}, G_{(1)}\right)\right)
$$

onde $J\left(x_{1}, G_{(1)}\right)=\left(g_{y x_{2}}, g_{y y}\right)$.

De (1) e (2) temos:

$$
\mu_{(3)}(f)+1=\mu_{(2)}(f)-\mu_{(1)}(f)+\mu_{(2)}\left(f^{\prime}\right)-\mu_{(1)}\left(f^{\prime}\right)
$$

e usando a hipótese de indução

$$
\mu_{(3)}(f)-\mu_{(2)}(f)+\mu_{(1)}(f)=m_{f}(0)-3-1 .
$$

(iii) A prova é novamente por indução sobre $n$. Vamos supor que a fórmula vale para $n-k_{s} \mathrm{e}$ vamos mostrar que vale para $n$. Os dois últimos números de Milnor na soma alternada do lado esquerdo de $F_{\left(k_{1}, \ldots, k_{s}\right)}(r)$ são números de Milnor de interseções completas que estão contidos em espaços de diferentes dimensões. Estes números de Milnor são relacionados como segue. Consideramos para $k_{1}+\ldots+k_{s-1}$ as multiplicidades locais das aplicações equidimensionais:

$$
m_{G_{\left(k_{1}, \ldots, k_{s-1}, 0\right)}}(0)=\left(m_{f}(0)-\sum_{i=1}^{s-1}\left(k_{i}+1\right)\right) m_{G_{\left(k_{1}, \ldots, k_{s-1}\right)}}(0) .
$$

Para os correspondentes números de Milnor segue da Proposição 3.1.1 que

$$
\mu_{\left(k_{1}, \ldots, k_{s-1}, 0\right)}=\left(m_{f}(0)-\sum_{i=1}^{s-1}\left(k_{i}+1\right)\right) \mu_{\left(k_{1}, \ldots, k_{s-1}\right)}+m_{f}(0)-\sum_{i=1}^{s-1}\left(k_{i}+1\right)-1
$$


onde $\sum_{i=1}^{s-1}\left(k_{i}+1\right)+1=k_{1}+\ldots+k_{s-1}+s$. Suponhamos $k_{s} \geq 1$. O passo de indução é então análogo ao caso $s=1$, porque os números de Milnor no lado esquerdo de $F_{\left(k_{1}, \ldots, k_{s}\right)}(r)$ - exceto o primeiro e o último - são aqueles das interseções completas da sequência encaixante

$$
\bar{A}_{\left(k_{1}, \ldots, k_{s-1}, k_{s}-1\right)} \subset \bar{A}_{\left(k_{1}, \ldots, k_{s-1}, k_{s}-2\right)} \subset \ldots \subset \bar{A}_{\left(k_{1}, \ldots, k_{s-1}, 0\right)} \subset \mathbb{C}^{n+s-1}, 0
$$

O primeiro e o segundo número de Milnor em $F_{\left(k_{1}, \ldots, k_{s}\right)}(r)$ são relacionados por

$$
\begin{gathered}
\mu_{\left(k_{1}, \ldots, k_{s}-1\right)}+\mu_{\left(k_{1}, \ldots, k_{s}-1\right)}^{\prime}= \\
\mu_{\left(k_{1}, \ldots, k_{s}\right)}+1+\operatorname{col}\left(\mathcal{I}\left(A_{\left(k_{1}, \ldots, k_{s}-1\right)}\right)+\mathcal{I}\left(M_{\left(k_{1}, \ldots, k_{s}-2\right)}\right)\right),
\end{gathered}
$$

Para $i=1, \ldots, k_{s}-2$ temos

$$
\begin{aligned}
\mu_{\left(k_{1}, \ldots, k_{s-1}, i\right)}+ & \mu_{\left(k_{1}, \ldots, k_{s-1}, i\right)}^{\prime}=\operatorname{col}\left(\mathcal{I}\left(A_{\left(k_{1}, \ldots, k_{s-1}, i+1\right)}\right)+\mathcal{I}\left(M_{\left(k_{1}, \ldots, k_{s-1}, i\right)}\right)\right) \\
& +\operatorname{col}\left(\mathcal{I}\left(A_{\left(k_{1}, \ldots, k_{s-1}, i\right)}\right)+\mathcal{I}\left(M_{\left(k_{1}, \ldots, k_{s-1}, i-1\right)}\right)\right) .
\end{aligned}
$$

Os dois últimos números de Milnor em $F_{\left(k_{1}, \ldots, k_{s}\right)}(r)$ são relacionados como segue

$$
\begin{gathered}
\mu_{\left(k_{1}, \ldots, k_{s-1}, 0\right)}+\mu_{\left(k_{1}, \ldots, k_{s-1}, 0\right)}^{\prime}=\operatorname{col}\left(\mathcal{I}\left(A_{\left(k_{1}, \ldots, k_{s-1}, 1\right)}\right)+\mathcal{I}\left(M_{\left(k_{1}, \ldots, k_{s-1}, 0\right)}\right)\right) \\
+\left(m_{f}(0)-\sum_{i=1}^{s-1}\left(k_{i}+1\right)\right)\left(\mu_{\left(k_{1}, \ldots, k_{s-1}\right)}+\mu_{\left(k_{1}, \ldots, k_{s-1}\right)}^{\prime}\right) .
\end{gathered}
$$

Tomando a soma alternada das fórmulas acima e trocando a soma alternada resultante dos $\mu^{\prime}$ por $m_{f}(0)-(n-1)-s$ (usando a fórmula $F_{\left(k_{1}, \ldots, k_{s}-1\right)}(r)$ em dimensão $\left.n-1\right)$ temos a fórmula $F_{\left(k_{1}, \ldots, k_{s}\right)}(r)$.

(ii) e (iv) Aqui temos as sequências encaixantes de inclusões para $s=1$

$$
\emptyset=\bar{A}_{(r)} \subset \bar{A}_{(r-1)} \neq \emptyset \subset \ldots \subset \bar{A}_{(1)}=\Sigma_{f} \subset \mathbb{C}^{n}, 0
$$

e para $s>1$

$$
\emptyset=\bar{A}_{\left(k_{1}, \ldots, k_{s-1}, t+1\right)} \subset \bar{A}_{\left(k_{1}, \ldots, k_{s-1}, t\right)} \neq \emptyset \subset \ldots \subset \bar{A}_{\left(k_{1}, \ldots, k_{s-1}, 0\right)} \subset \mathbb{C}^{n+s-1}, 0
$$

As fórmulas seguem por indução sobre $n \geq r-s$. Segue da Proposição 2.1.7 que

$$
\mu_{(r-1)}+\mu_{(r-1)}^{\prime}=0+\operatorname{col}\left(\mathcal{I}\left(A_{(r-1)}\right)+\mathcal{I}\left(M_{(r-2)}\right)\right)
$$

ou, para $s>1$,

$$
\mu_{\left(k_{1}, \ldots, k_{s-1}, t\right)}+\mu_{\left(k_{1}, \ldots, k_{s-1}, t\right)}^{\prime}=0+\operatorname{col}\left(\mathcal{I}\left(A_{\left(k_{1}, \ldots, k_{s-1}, t\right)}\right)+\mathcal{I}\left(M_{\left(k_{1}, \ldots, k_{s-1}, t-1\right)}\right)\right) .
$$


Fazendo a soma alternada das fórmulas acima o lado direito da expressão resultante é zero e o lado esquerdo é igual a soma do lado esquerdo da fórmula desejada e uma expressão identica onde $\mu^{\prime}$ substitui $\mu$, mas a expressão em $\mu^{\prime}$ é zero pela hipótese de indução.

Observação 3.1.3 O que acontece com as fórmulas do Teorema anterior quando um dos invariantes é infinito? Se $m_{f}(0)=\infty$ então o eixo dos $y$ corresponde a uma reta de pontos do tipo $A_{(n)}$ ( respectivamente $\left.A_{\left(k_{1}, \ldots, k_{s}\right)}\right)$, logo o invariante $\mu_{(n)}(f)$ ( respectivamente $\left.\mu_{\left(k_{1}, \ldots, k_{s}\right)}(f)\right)$ é infinito. Portanto as fórmulas valem. Observe que se $n=1$ temos $\mu_{(1)}(f)=\infty$ se, e somente se, $m_{f}(0)=\infty$. Suponhamos que $m_{f}(0)<\infty$ e $n>1$. Seja $X_{i}=\bar{A}_{(i)}$, para $s=1$, ou $\bar{A}_{\left(k_{1}, \ldots, k_{s-1}, i\right)}$, para $s>1$, e $X_{i}^{\prime}=X_{i} \cap\left\{x_{1}=0\right\}$. Consideramos as sequências de inclusões

$$
\ldots \subset X_{i+1} \subset X_{i} \subset X_{i-1} \subset \ldots
$$

Se $\mu\left(X_{i}\right)=\infty$ segue que $\mu\left(X_{i-1}\right)=\mu\left(X_{i+1}\right)=\infty$. Logo $m_{f}(0)=\infty$. Portanto se $m_{f}(0)$ for finita todos os número de Milnor são finitos.

O teorema estabelece as seguintes fórmulas para os invariantes 0-estáveis de germes de aplicações equidimensionais de coposto 1 (por exemplo, para $n=2$ e $s=1$ obtemos a conhecida fórmula $c(f)=\mu\left(\Sigma_{f}\right)+m_{f}(0)-2$ que relaciona o número de cúspides, o número de Milnor do conjunto crítico e a multiplicidade local de um germe $f$ do plano no plano, ver [11]). Como $m_{G_{\left(k_{1}, \ldots, k_{s}\right)}}(0)=\mu_{\left(k_{1}, \ldots, k_{s}\right)}(f)+1$ e $m_{G_{\left(k_{1}, \ldots, k_{s}\right)}}(0)=c \cdot r_{\left(k_{1}, \ldots, k_{s}\right)}(f)$ temos o seguinte:

Corolário 3.1.4 Seja $f: \mathbb{C}^{n}, 0 \rightarrow \mathbb{C}^{n}, 0, n \geq 1$, um germe de aplicação de coposto 1 com multiplicidade local $m_{f}(0)=r$. Então

(i) Se $s=1$ e $r \geq n+1$ temos

$$
r_{(n)}(f)=\mu_{(n-1)}(f)-\mu_{(n-2)}(f)+\ldots+(-1)^{n} \mu_{(1)}(f)+m_{f}(0)-n .
$$

(ii) Se $s=1$ e $r<n+1$ temos $r_{(n)}(f)=0$.

(iii) Se $s>1$ e $r \geq n+s$ temos

$$
\begin{gathered}
r_{\left(k_{1}, \ldots, k_{s}\right)}(f)=\frac{1}{c}\left(\mu_{\left(k_{1}, \ldots, k_{s}-1\right)}(f)-\mu_{\left(k_{1}, \ldots, k_{s}-2\right)}(f)+\ldots+(-1)^{k_{s}+1} \mu_{\left(k_{1}, \ldots, k_{s-1}, 0\right)}(f)\right. \\
\left.+(-1)^{k_{s}}\left(m_{f}(0)-\sum_{i=1}^{s-1}\left(k_{i}+1\right)\right) \mu_{\left(k_{1}, \ldots, k_{s-1}\right)}(f)+m_{f}(0)-n-s+1\right) .
\end{gathered}
$$

onde c denota a ordem do subgrupo de $S_{s}$ que fixa a partição $k_{1}+\ldots+k_{s}$ de $n$. 
(iv) Se $s>1$ e $r<n+s$ temos $r_{\left(k_{1}, \ldots, k_{s}\right)}(f)=0$.

Observação 3.1.5 Para germes de aplicações quase homogêneas $f$ podemos substituir os números de Milnor $\mu$ nas fórmulas acima pelos correspondentes números de Tjurina $\tau$ ou pelos invariantes $v$, pois $\mu, \tau$ e $v$ coincidem no caso de ICIS de dimensão positiva.

O próximo corolário é importante para obtermos as aplicações da próxima seção.

Corolário 3.1.6 Seja $f_{t}$ uma deformação de um germe de aplicação de coposto 1 $f: \mathbb{C}^{n}, 0 \rightarrow \mathbb{C}^{n}, 0, n \geq 2$, de multiplicidade local constante $m_{f_{t}}(0)=m_{f}(0)$. Se os números de Milnor $\mu_{\sigma, 0}\left(f_{t}\right)$ são constantes para todas as partições $\sigma$ de $n-1, n-3, \ldots, r(r=1$ para $n$ par e $r=2$ para $n$ impar) então os números de Milnor $\mu_{\sigma}\left(f_{t}\right)$ e $\mu_{\sigma, 0}\left(f_{t}\right)$ são constantes para todas as partições $\sigma$ de $m \leq n$ ( em particular, os números de Milnor de todos os tipos estáveis são constantes).

\section{Demonstração:}

Vamos fazer a demonstração para $m_{f}(0) \geq 2 n$ ( o caso $m_{f}(0)<2 n$ é análogo). Considerando $n=2 l$ ou $n=2 l+1$, a prova é por indução sobre $l$. Usando as fórmulas do Teorema 3.1 .2 temos que para $n=2$ a constância de $\mu_{(1,0)}$ implica na constância dos outros invariantes e para $n=3$ a constância de $\mu_{(2,0)}$ e de $\mu_{(1,1,0)}$ implica a constância dos outros invariantes. Consideremos $n \geq 4$ e suponhamos que a afirmação vale para dimensões menores. Seja $k_{1}+\ldots+k_{s}=n$ reescrevemos as fórmulas $F_{\left(k_{1}, \ldots, k_{s}\right)}(r)$ de modo que em cada lado da igualdades só aparecem termos positivos e ignoramos a constante $m_{f}(0)-n-s$.

Das fórmulas $F_{\left(k_{1}, \ldots, k_{s-1}, 1\right)}$ temos que a constância de $\mu_{\left(k_{1}, \ldots, k_{s-1}, 0\right)}$ implica na constância de $\mu_{\left(k_{1}, \ldots, k_{s-1}, 1\right)}(f)$ e $\mu_{\left(k_{1}, \ldots, k_{s-1}\right)}(f)$.

Para $k_{s} \geq 2$ a fórmula $F_{\left(k_{1}, \ldots, k_{s}\right)}$ está relacionada com a fórmula $F_{\left(k_{1}, \ldots, k_{s}-2\right)}$ em dimensão $n-2$ da seguinte maneira:

$$
\mu_{\left(k_{1}, \ldots, k_{s}\right)}(f)+\mu_{\left(k_{1}, \ldots, k_{s}-2\right)}(f) \cdots=\mu_{\left(k_{1}, \ldots, k_{s}-1\right)}(f)+\cdots
$$

Da hipótese de indução temos que a constância dos $\mu_{\sigma, 0}$ para todas as partições $\sigma$ de $n-3, n-5, \ldots$ implica (para $n-2$ ) na constância de todos os termos da fórmula. Este fato mais a constância dos $\mu_{\left(k_{1}, \ldots, k_{s}-1\right)}$ (que segue da constância de $\mu_{\left(k_{1}, \ldots, k_{s-1}, 0\right)}$ ) isto implica que todos os números de Milnor em dimensão $n$ são constantes.

Observação 3.1.7 O corolário anterior ainda é válido se considerarmos os invariantes de $f$ e substituirmos "constante" por "finito".

Similarmente à demonstração do Teorema 3.1 .2 obtemos a seguinte fórmula para os invariantes $v_{(i)}(f)=v\left(G_{(i)}\right)$

$$
v_{(n)}(f)-v_{(n-1)}(f)+\ldots+(-1)^{(n+1)} v_{(1)}(f)+\kappa=\operatorname{dim}_{\mathbb{C}} \mathcal{O}_{n} / \mathcal{I}\left(A_{(n+1)}\right),
$$


onde $\kappa$ é um "termo de correção" definido como segue.

Considere, na notação do Lema 2.1.8, o caso $v_{\sigma}, R=\mathcal{O}_{n+s+l-1}, A=\mathcal{I}\left(A_{\left(k_{1}, \ldots, k_{s}, 0_{l}\right)}\right)$, $B=\mathcal{I}\left(\bar{M}_{\left(k_{1}, \ldots, k_{s}, 0_{l}\right)}\right), l=0,1, C=\mathcal{I}\left(\bar{M}_{\left(k_{1}, \ldots, k_{s}-1\right)}, l=0\right.$, ou $C=J\left(G_{\left(k_{1}, \ldots, k_{s}\right)}\right), l=1$, e $h$ a derivada da última componente de $G_{\left(k_{1}, \ldots, k_{s}, 0_{l}\right)}$ com relação à última variável, e $\delta_{\left(k_{1}, \ldots, k_{s}, 0_{l}\right)}=$ $\operatorname{col}(A+C)-\operatorname{col}((A+B): h+C)$. Então, para $s=1$, o "termo de correção" citado acima é dado por

$$
\kappa=\delta_{\left(k_{1}\right)}-\delta_{\left(k_{1}-1\right)}+\ldots+(-1)^{k_{1}} \delta_{(2)},
$$

e para $s>1$,

$$
\kappa=\delta_{\left(k_{1}, \ldots, k_{s}\right)}-\delta_{\left(k_{1}, \ldots, k_{s}-1\right)}+\ldots+(-1)^{k_{s}} \delta_{\left(k_{1}, \ldots, k_{s-1}, 0\right)} .
$$

Proposição 3.1.8 Seja $f: \mathbb{C}^{n}, 0 \rightarrow \mathbb{C}^{n}, 0, n \geq 1$, um germe de aplicação de coposto 1 e assuma que para cada $v_{k(s, m), 0_{l}}(f)$ aparecendo nas fórmulas acima o correspondente conjunto $\bar{A}_{k(s, m), 0_{l}}$ é não vazio e é uma ICIS. Suponhamos que $v_{(k(s, n))}<\infty$, ou seja, todos os invariantes que aparecem nas fórmulas são finitos. Então, para qualquer $s>1, k_{s}>0$

$$
\begin{gathered}
v_{\left(k_{1}, \ldots, k_{s}\right)}(f)-v_{\left(k_{1}, \ldots, k_{s}-1\right)}(f)+\ldots+(-1)^{k_{s}} v_{\left(k_{1}, \ldots, k_{s-1}, 0\right)}(f)+\kappa+ \\
\left.(-1)^{k_{s}+1}\left(m_{f}-\sum_{i=1}^{s-1}\left(k_{i}+1\right)\right) v_{\left(k_{1}, \ldots, k_{s-1}\right)}(f)=\operatorname{col}\left(\mathcal{I}\left(A_{\left(k_{1}, \ldots, k_{s}+1\right.}\right)\right)+\mathcal{I}^{\prime}\right)
\end{gathered}
$$

e para $s=1, k_{1}>0$ temos

$$
\left.v_{\left(k_{1}\right)}(f)-v_{\left(k_{1}-1\right)}(f)+\ldots+(-1)^{k_{1}+1} v_{(1)}(f)+\kappa=\operatorname{col}\left(\mathcal{I}\left(A_{\left(k_{1}+1\right.}\right)\right)+\mathcal{I}^{\prime}\right),
$$

onde $\mathcal{I}^{\prime}=0$, para $\sum_{i=1}^{s} k_{i}=n$, e $\mathcal{I}^{\prime}=\mathcal{I}\left(\bar{M}_{\left(k_{1}, \ldots, k_{s}\right)}\right)$, para $\sum_{i=1}^{s} k_{i}<n$.

\section{Demonstração:}

Esta demonstração é similar à do Teorema 3.1.2. Seja $\mathcal{I}\left(\bar{M}_{\left(k_{1}, \ldots, k_{s}\right)}\right)$ o ideal gerado pelos determinantes das maiores submatrizes de $d G_{\left(k_{1}, \ldots, k_{s}\right)}$ com a última coluna, a coluna $\partial / \partial \epsilon_{s}$ (para $s>1$ ) ou a coluna $\partial / \partial y($ para $s=1)$, omitida (para $\left.\sum_{i=1}^{s} k_{i}<n\right)$.

Sejam $v_{k(s, m)}:=v_{k(s, m)}(f)$ e $h$ a última função componente de $G_{\left(k_{1}, \ldots, k_{s}+1\right)}$. Para $k_{s}>0$ e $\sum_{i} k_{i}=2, \ldots, n-1$ temos

$$
\begin{aligned}
& v_{\left(k_{1}, \ldots, k_{s}\right)}=\operatorname{col}\left(\mathcal{I}\left(A_{\left(k_{1}, \ldots, k_{s}\right)}\right)+h \mathcal{I}\left(\bar{M}_{\left(k_{1}, \ldots, k_{s}-1\right)}\right)+\mathcal{I}\left(\bar{M}_{\left(k_{1}, \ldots, k_{s}\right)}\right)\right) \\
& =\operatorname{col}\left(\mathcal{I}\left(A_{\left(k_{1}, \ldots, k_{s}+1\right)}\right)+\mathcal{I}\left(\bar{M}_{\left(k_{1}, \ldots, k_{s}\right)}\right)\right) \\
& \quad+\operatorname{col}\left(\mathcal{I}\left(A_{\left(k_{1}, \ldots, k_{s}\right)}\right)+\mathcal{I}\left(\bar{M}_{\left(k_{1}, \ldots, k_{s}-1\right)}\right)\right)-\delta_{\left(k_{1}, \ldots, k_{s}\right)}
\end{aligned}
$$

Para $k_{s}>0$ e $\sum_{i} k_{i}=n$ temos

$$
v_{\left(k_{1}, \ldots, k_{s}\right)}=\operatorname{col}\left(\mathcal{I}\left(A_{\left(k_{1}, \ldots, k_{s}\right)}\right)+h \mathcal{I}\left(\bar{M}_{\left(k_{1}, \ldots, k_{s}-1\right)}\right)\right)=
$$




$$
\operatorname{col}\left(\mathcal{I}\left(A_{\left(k_{1}, \ldots, k_{s}+1\right)}\right)\right)+\operatorname{col}\left(\mathcal{I}\left(A_{\left(k_{1}, \ldots, k_{s}\right)}\right)+\mathcal{I}\left(\bar{M}_{\left(k_{1}, \ldots, k_{s}-1\right)}\right)\right)-\delta_{\left(k_{1}, \ldots, k_{s}\right)},
$$

e para $s=1$ e $k_{1}=1$ obtemos $v_{(1)}=\operatorname{col}\left(\mathcal{I}\left(A_{(2)}\right)+\mathcal{I}\left(\bar{M}_{(1)}\right)\right)$.

Finalmente, para $k_{s}=0, s>1$, os invariantes $v_{\left(k_{1}, \ldots, k_{s-1}, 0\right)}, v_{\left(k_{1}, \ldots, k_{s-1}, 1\right)}$ e $v_{\left(k_{1}, \ldots, k_{s-1}\right)}$ são relacionados como segue:

$$
\begin{aligned}
& v_{\left(k_{1}, \ldots, k_{s-1}, 0\right)}=\operatorname{col}\left(\mathcal{I}\left(A_{\left(k_{1}, \ldots, k_{s-1}, 1\right)}\right)+\mathcal{I}\left(\bar{M}_{\left(k_{1}, \ldots, k_{s-1}, 0\right)}\right)\right) \\
& +\operatorname{col}\left(\mathcal{I}\left(A_{\left(k_{1}, \ldots, k_{s-1}, 0\right)}\right)+J\left(G_{\left(k_{1}, \ldots, k_{s-1}\right)}\right)\right)-\delta_{\left(k_{1}, \ldots, k_{s-1}, 0\right)},
\end{aligned}
$$

onde $\left.J\left(G_{\left(k_{1}, \ldots, k_{s-1}\right)}\right)\right)$ é o ideal dos maiores menores de $d G_{\left(k_{1}, \ldots, k_{s-1}\right)}$.

O segundo termo no lado direito é o comprimento de um ideal em $\mathcal{O}_{n+s-1}$, que é da forma

$$
\mathcal{I}\left(A_{\left(k_{1}, \ldots, k_{s-1}\right)}\right)+J\left(G_{\left(k_{1}, \ldots, k_{s-1}\right)}\right)+(h),
$$

onde $h$ é um polinômio mônico em $\epsilon_{s}$ de grau $m_{f}-\sum_{i=1}^{s-1}\left(k_{i}+1\right)$ e as duas primeiras parcelas definem um ideal em $\mathcal{O}_{n+s-2}$ de comprimento finito, de fato de comprimento $v_{\left(k_{1}, \ldots, k_{s-1}\right)}<\infty$. Portanto,

$$
\operatorname{col}\left(\mathcal{I}\left(A_{\left(k_{1}, \ldots, k_{s-1}, 0\right)}\right)+J\left(G_{\left(k_{1}, \ldots, k_{s-1}\right)}\right)\right)=\left(m_{f}-\sum_{i=1}^{s-1}\left(k_{i}+1\right)\right) v_{\left(k_{1}, \ldots, k_{s-1}\right)}
$$

e $\log 0$

$$
\begin{aligned}
& v_{\left(k_{1}, \ldots, k_{s-1}, 1\right)}-v_{\left(k_{1}, \ldots, k_{s-1}, 0\right)}+\left(m_{f}-\sum_{i=1}^{s-1}\left(k_{i}+1\right)\right) v_{\left(k_{1}, \ldots, k_{s-1}\right)} \\
& =\operatorname{col}\left(\mathcal{I}\left(A_{\left(k_{1}, \ldots, k_{s-1}, 2\right)}\right)+\mathcal{I}\left(\bar{M}_{\left(k_{1}, \ldots, k_{s-1}, 1\right)}\right)\right)-\delta_{\left(k_{1}, \ldots, k_{s-1}, 0\right)} .
\end{aligned}
$$

Agora, tomando somas alternadas:

$$
v_{\left(k_{1}, \ldots, k_{s-1}, k_{s}\right)}-v_{\left(k_{1}, \ldots, k_{s-1}, k_{s}-1\right)}+\ldots+(-1)^{k_{s}+1}\left(m_{f}-\sum_{i=1}^{s-1}\left(k_{i}+1\right)\right) v_{\left(k_{1}, \ldots, k_{s-1}\right)}
$$

temos a fórmula para $s>1$. Finalmente, para $s=1$ temos $v_{\left(k_{1}\right)}-v_{\left(k_{1}-1\right)}+\ldots+(-1)^{k_{1}+1} v_{(1)}$.

Observação 3.1.9 (i) Apesar dos termos de correção, as fórmulas acima relacionando os invariantes $v$ implicam a finitude de certos invariantes. Por exemplo, para $n=3$ a finitude de $v_{(1,1)}(f)$ e de $v_{(3)}(f)$ implica a finitude de $v_{(1,2)}(f)$. Seja $B_{\left(k_{1}, k_{2}\right)}=\operatorname{col}\left(\mathcal{I}\left(A_{\left(k_{1}, k_{2}\right)}\right)+\mathcal{I}\left(\bar{M}_{\left(k_{1}, k_{2}-1\right)}\right)\right)$. Então $v_{(1,1)}(f)<\infty$ e

$$
v_{(1,1)}(f)=B_{(1,2)}+B_{(1,1)}-\delta_{(1,1)}
$$

implica $B_{(1,2)}<\infty$. Como $v_{(3)}(f)<\infty$, obtemos $\operatorname{col}\left(\mathcal{I}\left(A_{(1,3)}\right)\right)<\infty$. Agora

$$
v_{(1,2)}(f)=\operatorname{col}\left(\mathcal{I}\left(A_{(1,3)}\right)\right)+B_{(1,2)}-\delta_{(1,2)},
$$


implica $v_{(1,2)}(f)<\infty$, como queríamos. (Por outro lado, a fórmula $F_{(3)}$ para os números de Milnor vale para $f \mathcal{K}$-finitamente determinado que $v_{(3)}(f)$ e $v_{(1)}(f)$ são finitos se $v_{(2)}(f)$ é finito. Isto é difícil de mostrar para $v$ diretamente, mas é claro que $v_{(i)}(f)$ é finito se, e somente se, $\mu_{(i)}(f)$ é finito.)

(ii) Consideramos os conjuntos $\bar{A}_{\left(k_{1}, \ldots, k_{s-1}, 0\right)} \subset \mathbb{C}^{n+s-1}$ consistindo de $s-1$ pontos singulares $p_{i}$ (do tipo $A_{\geq k_{i}}$ ) e um ponto regular $p_{s}$ tendo a mesma imagem por $f$. Se

$$
\mathcal{I}=\mathcal{I}\left(A_{\left(k_{1}, \ldots, k_{s-1}, 0\right)}\right)+J\left(G_{\left(k_{1}, \ldots, k_{s-1}, 0\right)}\right)
$$

(o comprimento de $\mathcal{I}$ é $\left.v_{\left(k_{1}, \ldots, k_{s-1}, 0\right)}(f)\right)$ temos pela sequência exata

$$
0 \longrightarrow \mathcal{O}_{n+s-1} /\left(\mathcal{I}: \epsilon_{s}\right) \longrightarrow \mathcal{O}_{n+s-1} / \mathcal{I} \longrightarrow \mathcal{O}_{n+s-1} /\left(\mathcal{I}+\left(\epsilon_{s}\right)\right) \longrightarrow 0
$$

que $v_{\left(k_{1}, \ldots, k_{s-1}, 0\right)}(f)=v_{\left(k_{1}, \ldots, k_{s-1}+1\right)}(f)+\operatorname{col}\left(\mathcal{I}: \epsilon_{s}\right)$.

Portanto o invariante $v_{\left(k_{1}, \ldots, k_{s-1}, 0\right)}(f)$ tem uma diagonal e uma contribuição fora da diagonal. De modo geral, tomando

$$
\mathcal{I}=\mathcal{I}\left(A_{\left(k_{1}, \ldots, k_{s-1}, k_{s}\right)}\right)+J\left(G_{\left(k_{1}, \ldots, k_{s-1}, k_{s}\right)}\right)
$$

$\left(\operatorname{com} k_{s} \geq 0\right)$ obtemos da mesma forma $v_{\left(k_{1}, \ldots, k_{s-1}, k_{s}\right)}(f)=v_{\left(k_{1}, \ldots, k_{s-1}+k_{s}+1\right)}(f)+\operatorname{col}\left(\mathcal{I}: \epsilon_{s}\right)$.

Para germes de aplicações semi-quasehomogêneas temos o seguinte resultado.

Proposição 3.1.10 Seja $f: \mathbb{C}^{n}, 0 \rightarrow \mathbb{C}^{n}, 0$ um germe de aplicação semi quase homogênea e seja c o fator gerado pelas permutações que fixam uma dada partição $k(s, n)$ de comprimento $s \geq 2$, então:

$$
\begin{aligned}
r_{k(s, n)}(f) & =c^{-1} \prod_{i=n+1}^{n+s-1}\left(m_{f}(0)-i\right) r_{(n)}(f) \\
& =c^{-1} \prod_{i=n+1}^{n+s-1}\left(m_{f}(0)-i\right)\left(m_{f}(0)+\sum_{j=1}^{n-1}(-1)^{j+1} \mu_{(n-j)}(f)-n\right) .
\end{aligned}
$$

(Mais geralmente, se $f$ tem uma decomposição $f=f_{0}+f_{+}$com $f_{0}$ e os correspondentes $G_{k(s, m)}$, para $k(s, m)$ aparecendo em ambos os lados das fórmulas, $\mathcal{K}$-finitamente determinados então as fórmulas ainda são válidas.)

Demonstração: Uma deformação de uma germe semi-quase-homogêneo $f=f_{0}+f_{+}$para $f_{0}$ induz deformações simultaneas das aplicações $G_{k(s, m)}$ as suas partes iniciais $\mathcal{K}$-finitamente determinados, que são topologicamente triviais por um resultado de Damon [6]. (Alternativamente, isto segue diretamente do fato das partes iniciais de $G_{k(s, m)}$ serem $\mathcal{K}$-finitamente determinadas.) E os números de Milnor de $G_{k(s, m)}$ e a multiplicidade local $m_{f}(0)$ envolvidas 
na fórmula são invariantes topológicos. Isto é então suficiente para provar a fórmula no caso semi-quase-homogêneo.

Consideremos a pré-forma normal para um germe quase-homogêneo equidimensional de coposto 1

$$
f: \mathbb{C}^{n}, 0 \rightarrow \mathbb{C}^{n}, 0, \quad(\mathbf{x}, y) \mapsto(\mathbf{x}, g(\mathbf{x}, y))=\left(\mathbf{x}, y^{k}+P_{1}(\mathbf{x}) y+\ldots+P_{k-2}(\mathbf{x}) y^{k-2}\right)
$$

com pesos $w_{1}, \ldots, w_{n-1}, 1$ para $x_{1}, \ldots, x_{n-1}, y$ e graus pesados $\delta_{1}=w_{1}, \ldots, \delta_{n-1}=w_{n-1}, \delta_{n}=$ $m_{f}(0)$. Usando os mesmos pesos $w_{i}$, para $i=1, \ldots, n$, como para $f$, e colocando nas variáveis adicionais $\epsilon_{j}$ peso 1 as aplicações

$$
G_{k(s, n)}=\left(G_{1}, \ldots, G_{n+s-1}\right): \mathbb{C}^{n+s-1}, 0 \rightarrow \mathbb{C}^{n+s-1}
$$

tem graus pesados

$$
m_{f}(0)-1, m_{f}(0)-2, \ldots, m_{f}(0)-(n+s-1) .
$$

A primeira igualdade na proposição segue quando expressamos as multiplicidades locais nas fórmulas $r_{k(s, n)}(f)=c^{-1} m_{G_{k(s, n)}}(0)$ e $r_{(n)}(f)=m_{G_{(n)}}(0)$ por pesos e graus pesados (usando a fórmula de Bezout generalizada) e comparando as expressões resultantes.

Observação 3.1.11 A hipótese de semi-quase-homogeneidade de $f$ na Proposição 3.1 .10 é necessária: os germes $f_{k}=\left(x, x y^{2}+y^{4}+x^{k} y\right)$ não são semi-quase-homogêneos para $k \geq 2 \mathrm{e}$ tem número de pontos de dobra $r_{(1,1)}\left(f_{k}\right)=d\left(f_{k}\right)=k$, mas $m_{f_{k}}(0)=4$ e $r_{(2)}\left(f_{k}\right)=c\left(f_{k}\right)=3$. 


\section{Capítulo 4}

\section{Aplicações}

\subsection{Germes de $\mathbb{C}^{n}$ em $\mathbb{C}^{p}, n>p$.}

Para germes de coposto 1 de $\mathbb{C}^{n}$ em $\mathbb{C}^{p}$ com $n<p$ as dimensões dos conjuntos $\bar{A}_{(i-1)}$ e $\bar{A}_{(i)}$ diferem por mais do que 1 (na verdade, por $p-n+1$ ). Portanto é improvável que existam fórmulas como somas alternadas de números de Milnor como as que encontramos no Teorema 3.1.2. Para germes de coposto $n-p+1$ ( isto é, minímo ) em dimensões $(n, p)$, onde $n \geq p$, as fórmulas no Teorema 3.1 .2 provavelmente se generalizam.

As fórmulas para germes de coposto 1 equidimensionais também são válidas para germes $\mathcal{A}$ simples $f: \mathbb{C}^{n} \rightarrow \mathbb{C}^{p}, n>p$, de posto $p-1$ se trocamos a mulplicidade local $m_{f}(0)$ por $\mu(f)+1$, pois observamos que um tal germe $f$ é dado pela pré-forma normal $\left(x, g(x, y)+\sum_{i=1}^{n-p} z_{i}^{2}\right)$, onde $(x, g(x, y))$ define um germe $\mathcal{A}$-simples equidimensional $\mathbb{C}^{p} \rightarrow \mathbb{C}^{p}$ de coposto 1 , ver [30], Lema 4.1. As fórmulas que valem para $(x, g(x, y))$ são as mesmas para $\left(x, g(x, y)+\sum_{i=1}^{n-p} z_{i}^{2}\right)$, pois os conjuntos $\bar{A}_{(i)}$ não se alteram.

A condição extra do germe ser $\mathcal{A}$-simples provavelmente não é necessária, por exemplo para $p=2$ e $s=1$, temos a seguinte:

Proposição 4.1.1 Seja $f: \mathbb{C}^{n}, 0 \rightarrow \mathbb{C}^{2}, 0, n \geq 2$, um germe de posto 1 . Então

$$
\mu_{(2)}(f)-\mu_{(1)}(f)=\mu(f)-2, \quad r_{(2)}(f)=\mu(f)+\mu_{(1)}(f)-1 .
$$

Observamos que para $n=2$ temos $\mu(f)=m_{f}(0)-1$, então reestabelecemos as fórmulas do Teorema 3.1.2 e Corolário 3.1.4 no caso $n=p=2, s=1$.

\section{Demonstração:}

Consideramos um germe $f: \mathbb{C}^{n}, 0 \rightarrow \mathbb{C}^{2}, 0$ de posto 1 dado pela pré-forma normal $f=\left(x, g\left(x, y_{1}, \ldots, y_{n-1}\right)\right)$ e sejam $g_{i}=\frac{\partial g}{\partial y_{i}}$ e $g_{i j}=\frac{\partial^{2} g}{\partial y_{i} \partial y_{j}}$. Então

$$
\mu_{(1)}(f)+\mu_{(1)}^{\prime}(f)=\operatorname{col}\left(\left(g_{1}, \ldots, g_{n-1}, \operatorname{det}\left(g_{i j}\right)_{1 \leq i, j \leq n-1}\right)\right)=r_{(2)}(f)=\mu_{(2)}(f)+1 .
$$

Além disso, $\mu_{(1)}^{\prime}(f)=\operatorname{col}\left(\left(x, g_{1}, \ldots, g_{n-1}\right)\right)-1$, onde

$$
\operatorname{col}\left(\left(x, g_{1}, \ldots, g_{n-1}\right)\right)=\mu\left(g\left(0, y_{1}, \ldots, y_{n-1}\right)\right)=\mu(f)
$$


(note que $f$ é $\mathcal{K}$-equivalente a uma suspensão de $g\left(0, y_{1}, \ldots, y_{n-1}\right)$ ).

Exemplo 4.1.2 Considere a série de germes $\mathcal{A}$-unimodais na classificação de Rieger em [28] dada por

$$
f_{k}=\left(x, x y+y^{3}+z^{3}+y^{k} z\right), \quad k \geq 3 .
$$

Os $f_{k}$ estão na $\mathcal{K}$-órbita de $D_{4}$, portanto $\mu\left(f_{k}\right)=4$, e $\mu_{(1)}\left(f_{k}\right)=k-1$. Portanto concluímos da segunda fórmula que $r_{(2)}\left(f_{k}\right)=k+2$, o que confere com o número de cúspides calculado em [28].

\subsection{Determinação finita e equisingularidade}

Agora consideramos aplicações das fórmulas na Seção 3.1 para a determinação finita. Lembramos que $\bar{A}_{\sigma}$, onde $\sigma$ é uma partição de $m \leq n$, é uma ICIS se, e somente se, $\mu_{\sigma}(f)<\infty$ ( ou equivalentemente, $v_{\sigma}(f)<\infty$ ).

De [26] sabemos que um germe $\mathcal{K}$-finitamente determinado $f: \mathbb{C}^{n}, 0 \rightarrow \mathbb{C}^{n}, 0$ de coposto 1 é $\mathcal{A}$-finitamente determinado se, e somente se, $v_{\sigma}(f)<\infty$ para todas as partições $\sigma$ de $m=2, \ldots, n$ com parcelas em $\{1,2\}$. Como consequência imediata do Corolário 3.1.6 temos o seguinte resultado.

Proposição 4.2.1 Seja $f: \mathbb{C}^{n}, 0 \rightarrow \mathbb{C}^{n}, 0$ um germe de coposto $1 \mathcal{K}$-finitamente determinado de multiplicidade local $m_{f}(0) \geq 2 n$. Então $f$ é $\mathcal{A}$-finitamente determinado se, e somente se, os conjuntos $\bar{A}_{\sigma, 0}$ são interseções completas com (pelo menos) a origem como ponto singular isolado para todas as partiçôes $\sigma$ de $n-1, n-3, \ldots, r(r=1$ para $n$ par e $r=2$ para $n$ impar $)$.

Observamos que o critério para $\mathcal{A}$-finitude de $f$ em [26] envolve somente o conjunto de pontos múltiplos $\bar{A}_{\sigma}$ na fonte, e não envolve os conjuntos de pontos múlitplos $\bar{A}_{\sigma, 0}$ correspondentes aos pontos regulares e singulares. Usando as fórmulas do Teorema 3.1 .2 (ou alternativamente as fórmulas da Proposição 3.1.8, veja Observação 3.1.9, parte (i)) também podemos simplificar o critério em [26]. Por exemplo, para $n=3$ podemos eliminar a condição de finitude em $v_{(1,2)}(f)$ em [26] e obter o seguinte resultado.

Proposição 4.2.2 Seja $f: \mathbb{C}^{3}, 0 \rightarrow \mathbb{C}^{3}, 0$ um germe de coposto $1 \mathcal{K}$-finitamente determinado. Então f é $\mathcal{A}$-finitamente determinado se, e somente se, os conjuntos $\bar{A}_{(2)}, \bar{A}_{(1,1)}$ e $\bar{A}_{(1,1,1)}$ são interseções completas com (pelo menos) a origem como ponto singular isolado ou são vazios.

\section{Demonstração:}

Usando as fórmulas $F_{(3)}(r)$ e $F_{(1,2)}(r)$ do Teorema 3.1 .2 para um germe de aplicação $f$ de multiplicidade local finita $r$ vemos que a hipótese da proposição implica que $\mu_{(1,2)}(f)<\infty$, e portanto, $v_{(1,2)}(f)<\infty$, ou $\bar{A}_{(1,2)}=\emptyset$. 
Outro critério para $f$ ser $\mathcal{A}$-finitamente determinado é dado a seguir.

Proposição 4.2.3 Seja $f: \mathbb{C}^{n}, 0 \rightarrow \mathbb{C}^{n}, 0$ um germe de coposto 1 de multiplicidade local $m_{f}(0)$. Então $f$ é $\mathcal{A}$-finitamente determinado se, e somente se, para $l=2, \ldots, \min \left(m_{f}(0), 2 n\right)$, os conjuntos $\bar{A}_{0_{l}}$ são interseções completas com (pelo menos) a origem como ponto singular isolado.

\section{Demonstração:}

As condições no conjunto $\bar{A}_{0_{l}}$ implicam que eles são não vazios e que $v_{0_{l}}(f)<\infty$. Usando a parte (ii) da Observação 3.1.9, temos que $v_{\sigma}(f)<\infty$ para todas as partições $\sigma$ de $m \leq n$ correspondentes a conjuntos não vazios $\bar{A}_{\sigma}$, e isto é equivalente ao fato de $f$ ser $\mathcal{A}$-finitamente determinado.

Reciprocamente, para $m_{f}(0)>2 n$ podemos obter $v_{\sigma}(f)<\infty$ para todas as partições $\sigma$ de $m \leq n$ para $v_{0_{l}}(f)<\infty, l=2, \ldots, 2 n$.

As aplicações mais interessantes do Teorema 3.1.2 são as que envolvem trivialidade topológica e equisingularidade de famílias de germes de coposto 1 de $\mathbb{C}^{n}$ em $\mathbb{C}^{n}$.

Observe, por exemplo, que o Corolário 3.1.6 também implica a excelência da deformação $f_{t}$ no sentido de [10]. Para $n=2$ temos:

Proposição 4.2.4 Seja $f_{t}$ uma deformação de um germe $f: \mathbb{C}^{2}, 0 \rightarrow \mathbb{C}^{2}, 0$ de coposto 1 com multiplicidade constante $m_{f_{t}}(0)=m_{f}(0)$, então temos o seguinte:

(i) A constância de $\mu_{(1,0)}\left(f_{t}\right)$ implica na constância dos números de Milnor de todos os tipos estáveis (isto é, $\mu_{\sigma}\left(f_{t}\right)$ é constante para todas as partições $\sigma$ de $m=1,2$ ).

(ii) $\mu_{(1,0)}\left(f_{t}\right)$ é constante se, e somente se, $f_{t}$ é Whitney equisingular (ao longo do eixo de parâmetros). E a Whitney equisingularidade de $f_{t}$ implica a trivialidade topológica.

\section{Demonstração:}

(i) Segue do Corolário 3.1.6 e do fato de germes de multiplicidade menor ou igual a 2 serem dobras ou difeomorfismos.

(ii) Segue de $(i)$ e da equivalência entre a Whitney equisingularidade de $f_{t}$ e a constância de $c\left(f_{t}\right)=r_{(2)}\left(f_{t}\right)$ e $d\left(f_{t}\right)=r_{(1,1)}\left(f_{t}\right)$, ver [11]. 
Observação 4.2.5 Em [7] Damon e Mond definem o número de Milnor do discriminante, $\mu_{\Delta}(f)$, de um germe $f: \mathbb{C}^{n}, 0 \rightarrow \mathbb{C}^{p}, 0, n \geq p$, como o número de $(p-1)$-esferas no discriminante de uma estabilização de $f$.

Para um germe $f: \mathbb{C}^{2}, 0 \rightarrow \mathbb{C}^{2}, 0$ temos de [7](usando a notação da tese) que

$$
\mu_{\Delta}(f)=\mu_{(1)}(f)+r_{(1,1)}(f)
$$

e para $n=3$ segue de [17] que

$$
\mu_{\Delta}(f)=\mu_{(1)}(f)+\frac{1}{2}\left(\mu_{(1,1)}(f)+\mu_{(3)}(f)\right)+r_{(1,1,1)}(f) .
$$

Portanto, suponhamos que $f_{t}$ é uma deformação de $f$ satisfazendo as condições do Corolário 3.1.6. então $\mu_{\Delta}\left(f_{t}\right)$ é constante. Por outro lado, para $n=2$ e 3 , a constância de $\mu_{\Delta}\left(f_{t}\right)$ e $m_{f_{t}}(0)$ implicam $\mu_{\sigma}\left(f_{t}\right)$ constante para todos os tipos estáveis $\sigma$ (isto segue das fórmulas para $\mu_{\Delta}(f)$ acima e das fórmulas do Teorema 3.1.2. 


\section{Capítulo 5}

\section{Classificação de germes de aplicações de $\mathbb{C}^{2}$ em $\mathbb{C}^{n}, n \geq 5$}

\subsection{Classificação de germes de aplicações de $\mathbb{C}^{2}$ em $\mathbb{C}^{5}$}

Um grande número de classificações de germes $\mathcal{A}$-simples $f: \mathbb{K}^{n}, 0 \rightarrow \mathbb{K}^{p}, 0$, onde $\mathbb{K}=\mathbb{R}$ ou $\mathbb{C}$, podem ser encontrados na literatura.

Singularidades simples de curvas planas foram classificadas por J. W. Bruce e T. J. Gaffney [4], e singularidades simples de curvas espacias foram classificadas por C. G. Gibson and C. A. Hobbs [13]. Em [1] Arnol'd estendeu os resultados acima classificando singularidades simples de curvas em qualquer dimensão.

Em [24] D. Mond classificou os germes $\mathcal{A}$-simples $f: \mathbb{R}^{2}, 0 \rightarrow \mathbb{R}^{3}, 0$. Para germes $\mathcal{A}$-simples de $\mathbb{C}^{2}$ em $\mathbb{C}^{2}$ temos a classificação de J. H. Rieger em [25].

Os autores C.A. Hobbs e N.P. Kirk em [16], usaram teoria de transversal completa e obtiveram a $\mathcal{A}$-classificação de multigermes simples de $\mathbb{R}^{2}$ em $\mathbb{R}^{3}$. No caso complexo esta classificação foi obtida por R.G. Wik-Atique em [32].

Em [18] C. Klotz, O. Pop and J. H. Rieger apresentam a classificação dos germes $\mathcal{A}$ simples de $\mathbb{R}^{n}$ em $\mathbb{R}^{2 n}, n \geq 2$, e também as modificações que devem ser feitas para obter esta classificação no caso complexo. Com a classificação apresentada neste trabalho podemos obter a lista dos germes $\mathcal{A}$-simples de $\mathbb{C}^{2}, 0$ em $\mathbb{C}^{4}, 0$, como segue. 
Teorema 5.1.1 ( [18]) Todo germe $\mathcal{A}$-simples $f: \mathbb{C}^{2}, 0 \rightarrow \mathbb{C}^{4}, 0$ é $\mathcal{A}$-equivalente a um dos seguintes germes:

$$
\begin{aligned}
& (x, y, 0,0) \\
& \left(x, x y, y^{2}, y^{2 k+1}\right), k \geq 1 \\
& \left(x, y^{2}, y^{3}, x^{k} y\right), k \geq 2 \\
& \left(x, y^{2}, y^{3}+x^{k} y, x^{l} y\right), l>k \geq 2 \\
& \left(x, y^{2}, x^{2} y+y^{2 k+1}, x y^{3}\right), k \geq 2 \\
& \left(x, y^{2}, x^{2} y, y^{5}\right) \\
& \left(x, y^{2}, x^{3} y+y^{5}, x y^{3}\right) \\
& \left(x, x y, x y^{2}+y^{3 k+1}, y^{3}\right), k \geq 1 \\
& \left(x, x y, x y^{2}+y^{3 k+2}, y^{3}\right), k \geq 1 \\
& \left(x, x y+y^{3 k+2}, x y^{2}, y^{3}\right), k \geq 1 \\
& \left(x, x y, y^{3}, y^{4}\right) \\
& \left(x, x y, y^{3}, y^{5}\right) \\
& \left(x, x y+y^{3}, x y^{2}+y^{2 k+1}, y^{4}\right), k \geq 2 \\
& \left(x, x^{2} y+y^{4}+y^{5}, x y^{2}, y^{3}\right) \\
& \left(x, x^{2} y+y^{4}, x y^{2}, y^{3}\right)
\end{aligned}
$$

No que segue exibiremos alguns resultados sobre a classificação de germes $\mathcal{A}$-simples de $\mathbb{C}^{2}$ em $\mathbb{C}^{n}, n \geq 5$. Começamos com a classificação de germes $\mathcal{A}$-simples de $\mathbb{C}^{2}$ em $\mathbb{C}^{5}$ de multiplicidade 2 .

Teorema 5.1.2 Todo germe $\mathcal{A}$-simples $f: \mathbb{C}^{2}, 0 \rightarrow \mathbb{C}^{5}, 0$, de multiplicidade 2 , é $\mathcal{A}$-equivalente a um dos seguintes germes:

$$
\begin{aligned}
& \left(x, x y, y^{2}, y^{2 k+1}, 0\right), k \geq 1 \\
& \left(x, y^{2}, y^{3}, x^{k} y, 0\right), k \geq 2 \\
& \left(x, y^{2}, y^{3}+x^{k} y, x^{l} y, 0\right), l>k \geq 2 \\
& \left(x, y^{2}, x^{2} y+y^{2 k+1}, x y^{3}, 0\right), k \geq 2 \\
& \left(x, y^{2}, x^{2} y, x y^{3}, y^{2 k+1}\right), k \geq 2 \\
& \left(x, y^{2}, x^{2} y, y^{5}, 0\right) \\
& \left(x, y^{2}, x^{2} y+y^{5}, x y^{5}, y^{7}\right) \\
& \left(x, y^{2}, x^{2} y, x y^{5}, y^{7}\right) \\
& \left(x, y^{2}, x^{3} y+y^{5}, x y^{3}, 0\right) \\
& \left(x, y^{2}, x^{3} y, x y^{3}, y^{5}\right) \\
& \left(x, y^{2}, x^{3} y, x y^{3}, y^{7}\right) \\
& \left(x, y^{2}, x^{3} y+x y^{3}, y^{5}, x^{4} y\right) \\
& \left(x, y^{2}, x^{3} y, x^{2} y^{3}, y^{5}\right) \\
& \left(x, y^{2}, x y^{3}, x^{4} y, y^{5}\right)
\end{aligned}
$$


Para demonstrar o Teorema acima vamos utilizar o método da Tranversal Completa descrito a seguir.

Proposição 5.1.3 (Transversal Completa para Jatos) Sejam $f \in \mathcal{M}_{n} \mathcal{O}_{n, p}$ e $T \subset$ $H^{k+1}(n, p)$ tais que

$$
\mathcal{M}_{n}^{k+1} \mathcal{O}_{n, p} \subset T \mathcal{A}_{1} f+T+\mathcal{M}_{n}^{k+2} \mathcal{O}_{n, p}
$$

então todo $(k+1)$-jato $j^{k+1} g(0)$, com $j^{k} g(0)=j^{k} f(0)$, está na mesma $\mathcal{A}_{1}^{k+1}$-órbita de $j^{k+1} f(0)+$ $t$ para algum $t \in T$.

Segue alguns detalhes da prova da classificação.

Lema 5.1.4 Dado um germe $f: \mathbb{C}^{2}, 0 \rightarrow \mathbb{C}^{5}, 0$ de multiplicidade 2 então seu 2-jato é $\mathcal{A}^{2}$ equivalente a $\left(x, x y, y^{2}, 0,0\right)$ ou $\left(x, y^{2}, 0,0,0\right)$.

\section{Demonstração:}

Seja $(x, 0,0,0,0)$ o 1 -jato de $f$ em $J^{1}(2,5)$. Primeiro vamos encontrar $T$ usando o método da Tranversal Completa:

$$
\mathcal{M}_{2}^{2} \mathcal{O}_{2,5} \subset T \mathcal{A}_{1} f+T+\mathcal{M}_{2}^{3} \mathcal{O}_{2,5}
$$

onde

$$
T \mathcal{A}_{1} f=\mathcal{M}_{2}^{2}\left\{\left[\begin{array}{l}
1 \\
0 \\
0 \\
0 \\
0
\end{array}\right]\right\}+f^{*} \mathcal{M}_{5}^{2}\left\{e_{1}, \ldots, e_{5}\right\}
$$

onde $e_{i}=(0, \ldots, 1, \ldots, 0)$ com 1 na $i$-ésima coordenada e zero nas demais.

Logo, $T=\left\{x y \cdot e_{i}, y^{2} \cdot e_{i}, i=2, \ldots 5\right\}$. Então o 2 -jato de $f$ é equivalente a:

$$
\left(x, a_{1} x y+b_{1} y^{2}, a_{2} x y+b_{2} y^{2}, a_{3} x y+b_{3} y^{2}, a_{4} x y+b_{4} y^{2}\right)
$$

Se $a_{1} \neq 0$ então através de mudanças de coordenadas na meta removemos o termo $x y$ das 3 últimas coordenadas e obtemos $\left(x, a_{1} x y+b_{1} y^{2}, \overline{b_{2}} y^{2}, \overline{b_{3}} y^{2}, \overline{b_{4}} y^{2}\right)$. Se $\overline{b_{i}} \neq 0$, para algum $i=2,3,4$, removemos o termo $y^{2}$ da segunda coordenada e obtemos após mudanças apropriadas $\left(x, x y, y^{2}, 0,0\right)$. Se $\overline{b_{i}}=0$, para $i=2,3,4$, temos $\left(x, a_{1} x y+b_{1} y^{2}, 0,0,0\right)$, então podemos completar o quadrado na segunda coordenada e através de mudança de coordenada na fonte obtemos $\left(x, y^{2}, 0,0,0\right)$.

Os 2 -jatos $(x, x y, 0,0,0)$ e $(x, 0,0,0,0)$ dão origem a germes de multiplicidade maior que 2 . 
Lema 5.1.5 Todo germe cujo 2-jato é $\left(x, x y, y^{2}, 0,0\right)$ é $\mathcal{A}$-equivalente a

$$
f=\left(x, x y, y^{2}, y^{2 k+1}, 0\right)
$$

para algum $k \geq 1$. Além disso, $f$ é simples, para todo $k \geq 1$.

\section{Demonstração:}

Vamos calcular $T \subset H^{2 k+1}(2,5)$, para $k \geq 1$, satisfazendo

$$
\mathcal{M}_{2}^{2 k+1} \mathcal{O}_{2,5} \subset T \mathcal{A}_{1} f+T+\mathcal{M}_{2}^{2 k+2} \mathcal{O}_{2,5}
$$

onde

$$
T \mathcal{A}_{1} f=\mathcal{M}_{2}^{2}\left\{\left[\begin{array}{l}
1 \\
y \\
0 \\
0 \\
0
\end{array}\right],\left[\begin{array}{c}
o \\
x \\
2 y \\
0 \\
0
\end{array}\right]\right\}+f^{*} \mathcal{M}_{5}^{2}\left\{e_{1}, \ldots, e_{5}\right\}
$$

Logo, $T=\left\{y^{2 k+1} . e_{4}, y^{2 k+1} \cdot e_{5}\right\}$ e após mudanças de coordenadas temos que os possíveis $(2 k+1)-$ jatos são

1. $\left(\left(x, x y, y^{2}, y^{2 k+1}, 0\right)\right.$

2. $\left(x, x y, y^{2}, 0,0\right)$

O germe (1) é finitamente determinado, pois $T=\emptyset$ em $H^{n}(2,5)$, para todo $n \geq 2 k+2$.

Assim obtemos os germes $\left(x, x y, y^{2}, y^{2 k+1}, 0\right), \quad k \geq 1$.

Temos que

$$
\left\{\begin{array}{ccc}
x^{i} y^{j} \cdot e_{l} & i>0, j \geq 0 & l=1, \ldots, 5 \\
y^{2 i} \cdot e_{l} & i>0 & l=1, \ldots, 5 \\
y^{2 i+1} \cdot e_{l} & i>0 & l=1,2,3 \\
y^{2 i+1} \cdot e_{l} & i \geq k & l=4,5
\end{array}\right\} \subset T \mathcal{A} f
$$

Logo o desdobramento versal de $f$ é

$$
f_{u v}=\left(x, x y, y^{2}, y^{2 k+1}+\sum_{i=1}^{k-1} u_{i} y^{2 i+1}, \sum_{j=1}^{k-1} v_{j} y^{2 j+1}\right)
$$

Temos que $f_{u v}$ pertence às seguintes órbitas: $f_{2 k+1}, f_{2 k-1}, \ldots, f_{3}$ e imersão. Como são finitas órbitas, o germe é simples. 
Lema 5.1.6 Dado o 2-jato $\left(x, y^{2}, 0,0,0\right)$. Obtemos as seguintes $\mathcal{A}^{3}$-órbitas:

1. $\left(x, y^{2}, y^{3}, x^{2} y, 0\right), \mathcal{A}-\operatorname{cod}=7$

2. $\left(x, y^{2}, y^{3}+x^{2} y, 0,0\right), \mathcal{A}-\operatorname{cod}=9$

3. $\left(x, y^{2}, y^{3}, 0,0\right), \mathcal{A}-\operatorname{cod}=10$

4. $\left(x, y^{2}, x y^{2}, 0,0\right), \mathcal{A}-\operatorname{cod}=10$

5. $\left(x, y^{2}, 0,0,0\right), \mathcal{A}-\operatorname{cod}=13$

\section{Demonstração:}

Seja $T \subset H^{3}(2,5)$ satisfazendo

$$
\mathcal{M}_{2}^{3} \mathcal{O}_{2,5} \subset T \mathcal{A}_{1} f+T+\mathcal{M}_{2}^{4} \mathcal{O}_{2,5}
$$

onde

$$
T \mathcal{A}_{1} f=\mathcal{M}_{2}^{2}\left\{\left[\begin{array}{l}
1 \\
0 \\
0 \\
0 \\
0
\end{array}\right],\left[\begin{array}{c}
0 \\
2 y \\
0 \\
0 \\
0
\end{array}\right]\right\}+f^{*} \mathcal{M}_{5}^{2}\left\{e_{1}, \ldots, e_{5}\right\}
$$

Logo, $T=\left\{x^{2} y \cdot e_{i}, y^{3} \cdot e_{i}, \quad i=3,4,5\right\}$ e o 3 -jato de $f$ é equivalente a

$$
\left(x, y^{2}, a_{1} x^{2} y+b_{1} y^{3}, a_{2} x^{2} y+b_{2} y^{3}, a_{3} x^{2} y+b_{3} y^{3}\right) .
$$

Se $a_{1} \neq 0$ então através de mudanças de coordenadas na meta removemos o termo $x^{2} y$ das 2 últimas coordenadas e obtemos

$$
\left(x, y^{2}, a_{1} x^{2} y+b_{1} y^{3}, \overline{b_{2}} y^{3}, \overline{b_{3}} y^{3}\right)
$$

Se $\overline{b_{i}} \neq 0$, para algum $i=2,3$, removemos o termo $y^{3}$ da terceira coordenada e obtemos, após mudanças apropriadas, $\left(x, y^{2}, x^{2} y, y^{3}, 0\right)$.

Se $\overline{b_{i}}=0$, para $i=2,3$, obtemos $\left(x, y^{2}, x^{2} y+y^{3}, 0,0\right)$.

Se $\overline{b_{i}}=0$, para $i=2,3$, e $b_{1}=0$, obtemos $\left(x, y^{2}, x^{2} y, 0,0\right)$.

Se $b_{1} \neq 0$ então com mudanças de coordenadas na fonte e na meta removemos o termo $y^{3}$ das 2 últimas coordenadas e obtemos

$$
\left(x, y^{2}, a_{1} x^{2} y+b_{1} y^{3}, \overline{a_{2}} x^{2} y, \overline{a_{3}} x^{2} y\right)
$$

Se $\overline{a_{i}} \neq 0$, para algum $i=2,3$, obtemos, após mudanças apropriadas, $\left(x, y^{2}, y^{3}, x^{2} y, 0\right)$ (já obtido anteriormente).

Se $\overline{a_{i}}=0$, para $i=2,3$, temos $\left(x, y^{2}, x^{2} y+y^{3}, 0,0\right)$ (já obtido anteriormente). 
Se $\overline{a_{i}}=0, i=2,3$, e $a_{1}=0$, obtemos $\left(x, y^{2}, y^{3}, 0,0\right)$.

Também obtemos $\left(x, y^{2}, 0,0,0\right)$ quando todos os coeficientes são nulos.

O germe (1) é finitamente determinado e simples.

Lema 5.1.7 Todo germe cujo 3 -jato é $\left(x, y^{2}, y^{3}+x^{2} y, 0,0\right)$ é $\mathcal{A}$ - equivalente a

$$
\left(x, y^{2}, y^{3}+x^{2} y, x^{l} y, 0\right)
$$

para algum $l \geq 3$. Além disso, o germe é simples.

\section{Demonstração:}

Vamos calcular $T \subset H^{l+1}(2,5)$, para $l \geq 3$, satisfazendo

$$
\mathcal{M}_{2}^{l+1} \mathcal{O}_{2,5} \subset T \mathcal{A}_{1} f+T+\mathcal{M}_{2}^{l+2} \mathcal{O}_{2,5}
$$

onde

$$
T \mathcal{A}_{1} f=\mathcal{M}_{2}^{2}\left\{\left[\begin{array}{c}
1 \\
0 \\
2 x y \\
0 \\
0
\end{array}\right],\left[\begin{array}{c}
o \\
2 y \\
3 y^{2}+x^{2} \\
0 \\
0
\end{array}\right]\right\}+f^{*} \mathcal{M}_{5}^{2}\left\{e_{1}, \ldots, e_{5}\right\}
$$

Logo, $T=\left\{x^{l} y \cdot e_{4}, x^{l} y \cdot e_{5}\right\}$ e após mudanças de coordenadas temos que os possíveis $(l+1)$-jatos são

1. $\left(x, y^{2}, y^{3}+x^{2} y, x^{l} y, 0\right)$

2. $\left(x, y^{2}, y^{3}+x^{2} y, 0,0\right)$

O germe (1) é finitamente determinado, pois $T=\emptyset \mathrm{em} H^{l+2}(2,5)$, para todo $l \geq 3$.

Assim obtemos os germes $\left(x, y^{2}, y^{3}+x^{2} y, x^{l} y, 0\right), \quad l \geq 3$.

As $\mathcal{A}$-órbitas sobre 3 . são completamente determinadas pelas séries $\left(x, y^{2}, y^{3}, x^{k} y, 0\right), k \geq 3 \mathrm{e}$ $\left(x, y^{2}, y^{3}+x^{k} y, x^{l} y, 0\right), l>k \geq 3$. Podemos combinar a série $\left(x, y^{2}, y^{3}, x^{k} y, 0\right), k \geq 3$ com a órbita em 1 ., tomando $k \geq 2$. Da mesma forma agrupamos a série $\left(x, y^{2}, y^{3}+x^{k} y, x^{l} y, 0\right), l>k \geq 3$ com a série $\left(x, y^{2}, y^{3}+x^{2} y, x^{l} y, 0\right), l \geq 3$, tomando $l>k \geq 2$

Sobre o 3 -jato em 4. obtemos:

$4.1\left(x, y^{2}, x y^{2}, x y^{3}, 0\right), \mathcal{A}-\operatorname{cod}=10$

$4.2\left(x, y^{2}, x y^{2}, 0,0\right), \mathcal{A}$-cod $=12$ 
As órbitas sobre 4.1 dão origem às séries $\left(x, y^{2}, x^{2} y, x y^{3}, y^{2 k+1}\right), k \geq 2 \mathrm{e}$ $\left(x, y^{2}, x^{2} y+y^{2 k+1}, x y^{3}, 0\right), k \geq 2$.

Sobre o 4 -jato em 4.2 obtemos:

4.2.1 $\left(x, y^{2}, x y^{2}, y^{5}, 0\right), \mathcal{A}-\operatorname{cod}=12$

4.2.2 $\left(x, y^{2}, x y^{2}+y^{5}, 0,0\right), \mathcal{A}-\operatorname{cod}=14$

$4.2 .3\left(x, y^{2}, x y^{2}, 0,0\right), \mathcal{A}-\operatorname{cod}=15$

A órbita em 4.2.1 é 5-determinada.

Sobre o 5-jato em 4.2.2 obtemos:

4.2.2.1 $\left(x, y^{2}, x y^{2}+y^{5}, x y^{5}, 0\right), \mathcal{A}-\operatorname{cod}=14$

4.2.2.2(x, $\left.y^{2}, x y^{2}+y^{5}, 0,0\right), \mathcal{A}-\operatorname{cod}=15$

Sobre o 6-jato em 4.2.2.1 obtemos:

4.2.2.1.1 $\left(x, y^{2}, x y^{2}+y^{5}, x y^{5}, y^{7}\right), \mathcal{A}$-cod $=14$ germe simples

4.2.2.1.2 $\left(x, y^{2}, x y^{2}+y^{5}, x y^{5}+\alpha y^{7}, 0\right)$ germe com modalidade $f_{\alpha}$

4.2.2.1.3 $\left(x, y^{2}, x y^{2}+y^{5}, x y^{5}, 0\right)$ adjacente ao anterior e portando não simples

Volto ao 6-jato 4.2.2.2 e obtemos:

4.2.2.2.1 $\left(x, y^{2}, x y^{2}+y^{5}, y^{7}, 0\right)$ adjacente a $f_{\alpha}$

4.2.2.2.2 $\left(x, y^{2}, x y^{2}+y^{5}, 0,0\right)$

Logo obtemos órbitas não simples, que estão no fecho de $f_{\alpha}$. Sobre o 5 -jato em 4.2 .3 obtemos :

4.2.3.1 $\left(x, y^{2}, x y^{2}, x y^{5}, 0\right), \mathcal{A}-\operatorname{cod}=15$

4.2.3.2 $\left(x, y^{2}, x y^{2}, 0,0\right), \mathcal{A}-\operatorname{cod}=17$

Sobre o 6 -jato em 4.2.3.1 obtemos o germe simples $\left(x, y^{2}, x y^{2}, x y^{5}, y^{7}\right)$ e germes não simples. Sobre o 6-jato em 4.2.3.2 obtemos apenas germes não simples.

Sobre o 3 -jato em 5 . obtemos

$5.1\left(x, y^{2}, x^{3} y, x y^{3}, 0\right), \mathcal{A}-\operatorname{cod}=13$

$5.2\left(x, y^{2}, x^{3} y+x y^{3}, 0,0\right), \mathcal{A}-\operatorname{cod}=15$ 
$5.3\left(x, y^{2}, x^{3} y, 0,0\right), \mathcal{A}-\operatorname{cod}=16$

$5.4\left(x, y^{2}, x y^{3}, 0,0\right), \mathcal{A}-\operatorname{cod}=16$

$5.5\left(x, y^{2}, 0,0,0\right), \mathcal{A}-\operatorname{cod}=19$

Em 5.1 obtemos duas séries de germes:

$\left(x, y^{2}, x^{3} y, x y^{3}, y^{2 k+1}\right)$ simples para $k=2$ e $k=3$ e não simples para $k \geq 4$

$\left(x, y^{2}, x^{3} y+y^{2 k+1}, x y^{3}, 0\right)$ simples para $k=2$ e não simples para $k \geq 3$

Sobre o 4-jato em 5.2 obtemos

5.2.1 $\left(x, y^{2}, x^{3} y+x y^{3}, y^{5}, x^{4} y\right), \mathcal{A}$-cod $=15$ germe simples

5.2.2 $\left(x, y^{2}, x^{3} y+x y^{3}, y^{5}+\alpha x^{4} y, 0\right)$, germe com modalidade $g_{\alpha}$

A partir de então obtemos órbitas não simples, que estão no fecho de $g_{\alpha}$.

Sobre o 4 -jato em 5.3 obtemos

5.3.1 $\left(x, y^{2}, x^{3} y, x^{2} y^{3}, y^{5}\right), \mathcal{A}-\operatorname{cod}=16$

5.3.2 $\left(x, y^{2}, x^{3} y, x^{2} y^{3}+y^{5}, 0\right)$, adjacente a $g_{\alpha}$

Logo obtemos órbitas não simples, que estão no fecho de $g_{\alpha}$.

Sobre o 4-jato em 5.4 obtemos

5.4.1 $\left(x, y^{2}, x y^{3}, x^{4} y, y^{5}\right)$, germe simples

$5.4 .2\left(x, y^{2}, x y^{3}, x^{4} y+y^{5}, 0\right)$, adjacente a $g_{\alpha}$

Segue que obtemos órbitas não simples, que estão no fecho de $g_{\alpha}$.

Sobre o 4-jato em 5.5 obtemos

5.5.1 $\left(x, y^{2}, x^{4} y, x^{2} y^{3}, y^{5}\right)$, adjacente a $\left(x, y^{2}, x^{4} y, 0, x^{3} y+x y^{3}\right)$ que por sua vez esta no fecho de $g_{\alpha}$, portanto não simples. 
Os diagramas a seguir dão uma idéia da classificação que foi feita utilizando o método da tranversal completa, começamos com o 2 -jato $\left(x, y^{2}, 0,0,0\right)$.

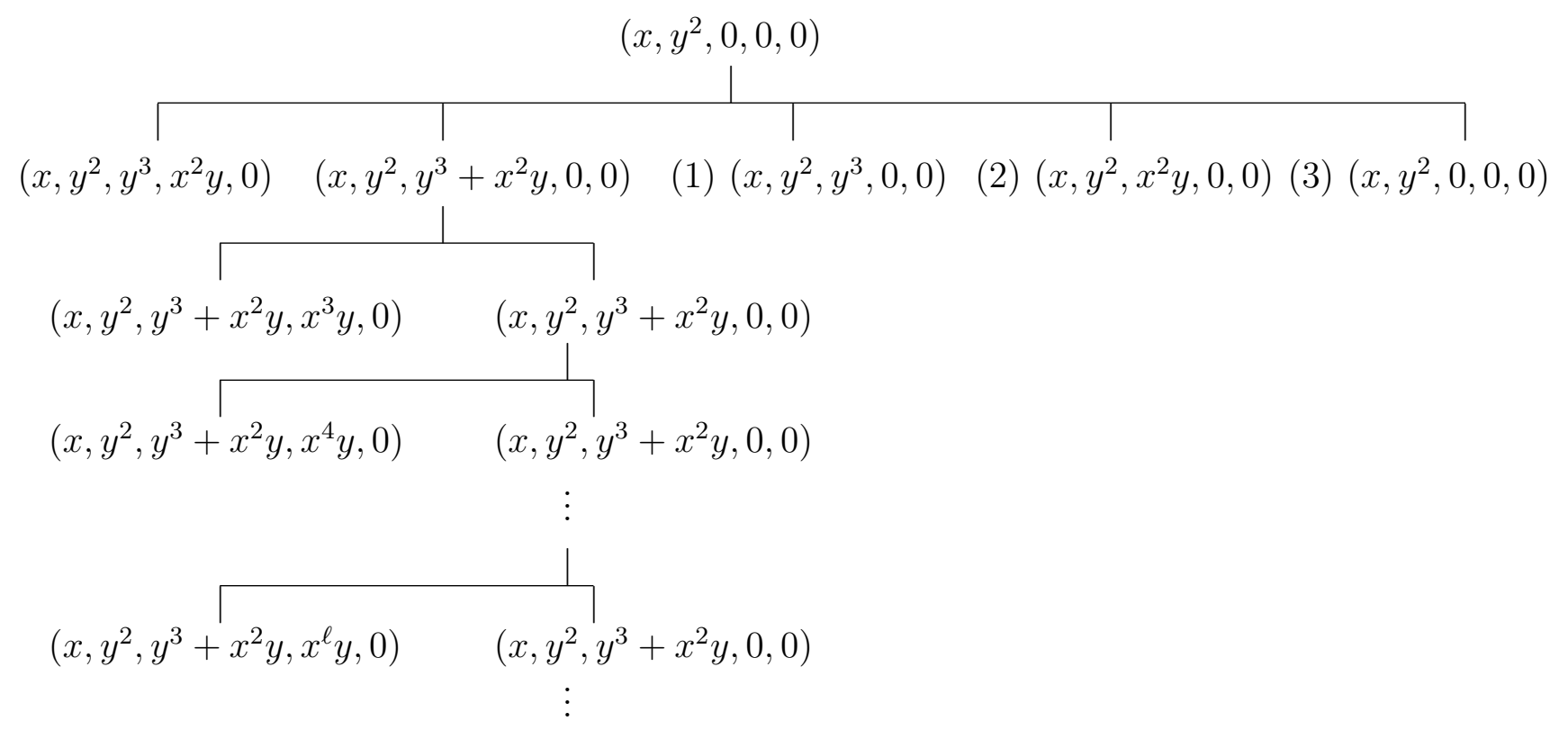

(1) $\left(x, y^{2}, y^{3}, 0,0\right)$

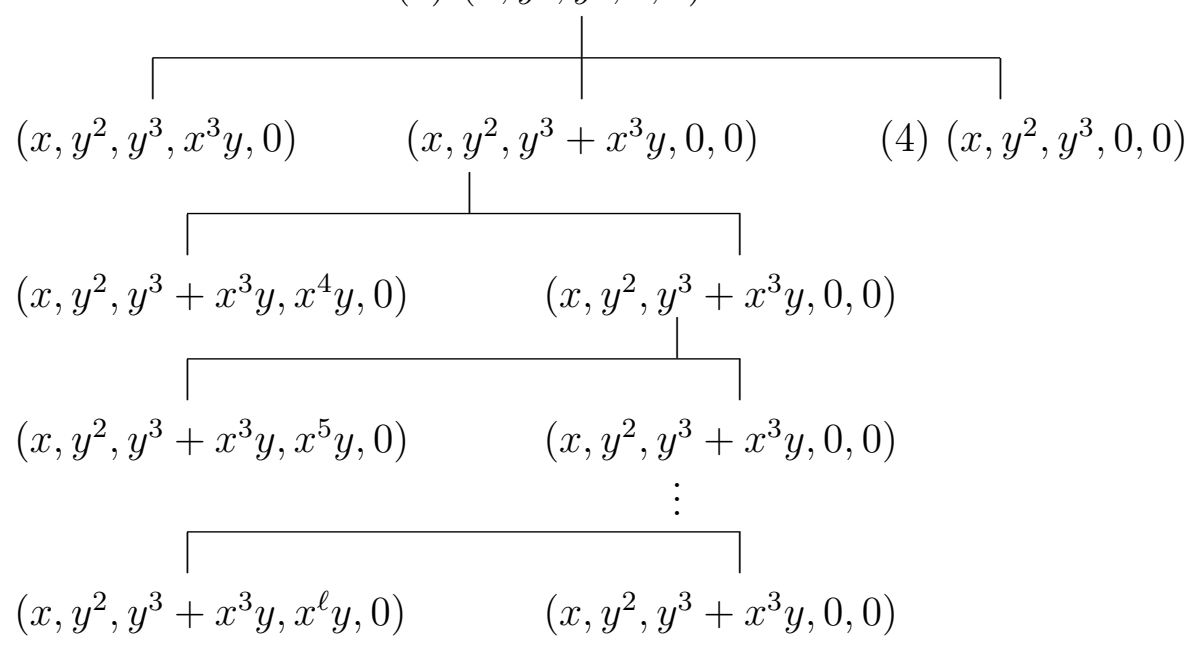




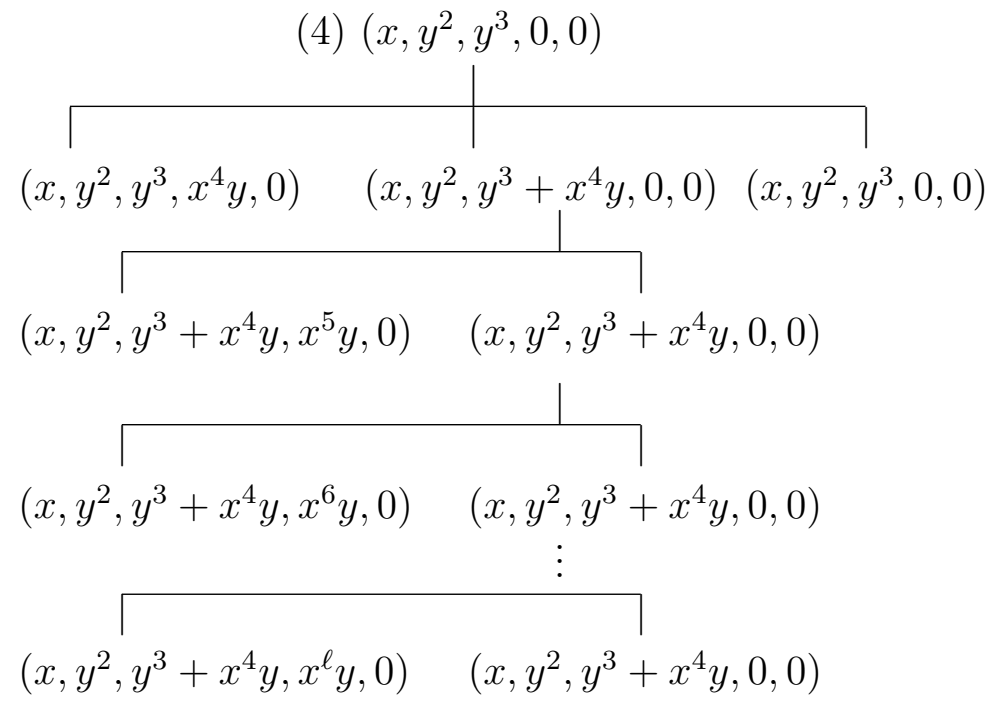

Encontramos as séries de germes simples:

$\left(x, y^{2}, y^{3}, x^{k} y, 0\right), k \geq 2$

$\left(x, y^{2}, y^{3}+x^{k} y, x^{\ell} y, 0\right), \ell>k \geq 2$

(2) $\left(x, y^{2}, x^{2} y, 0,0\right)$
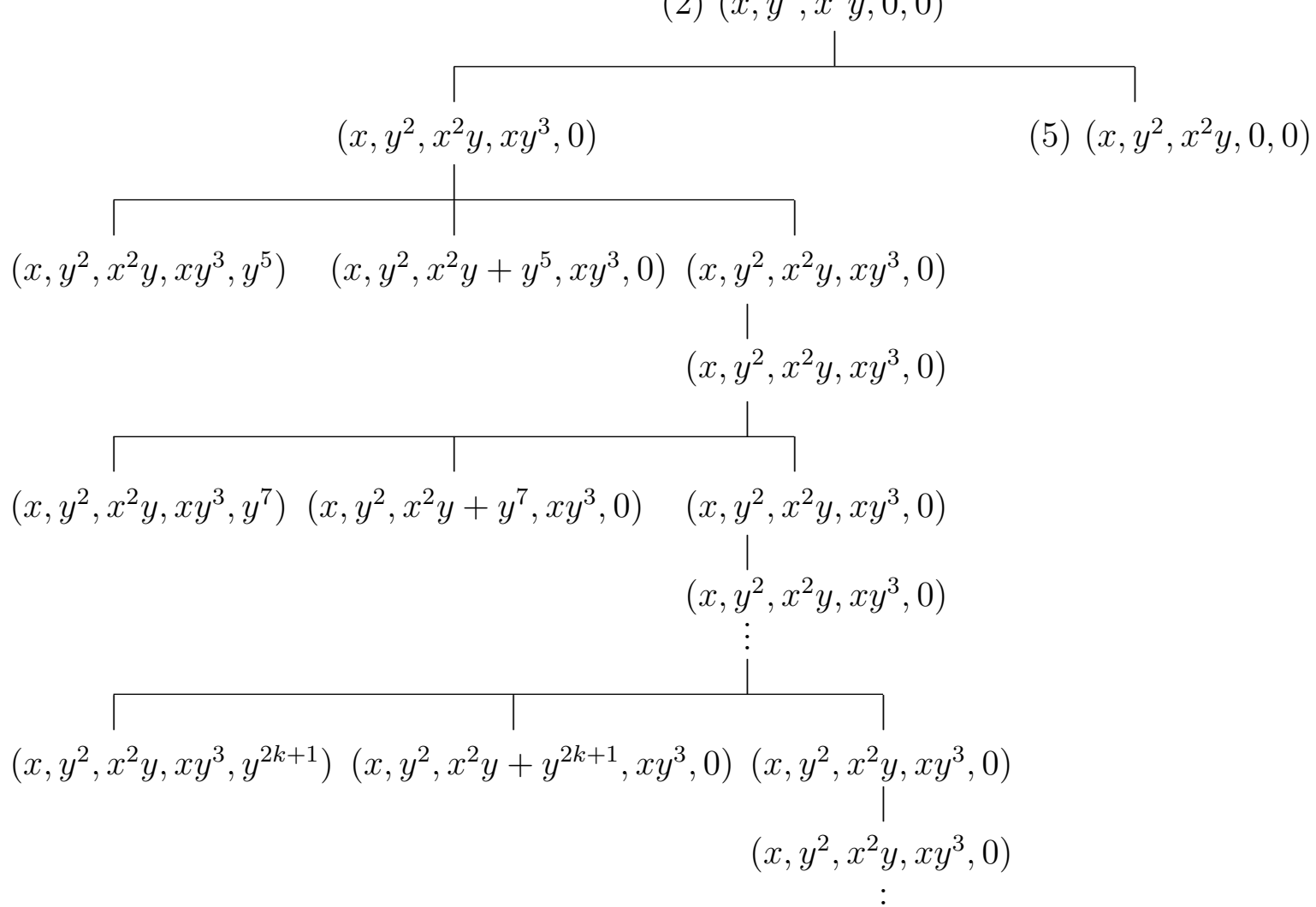

Encontramos as séries de germes simples:

$$
\begin{aligned}
& \left(x, y^{2}, x^{2} y, x y^{3}, y^{2 k+1}\right), k \geq 2 \\
& \left(x, y^{2}, x^{2} y+y^{2 k+1}, x y^{3}, 0\right), k \geq 2
\end{aligned}
$$



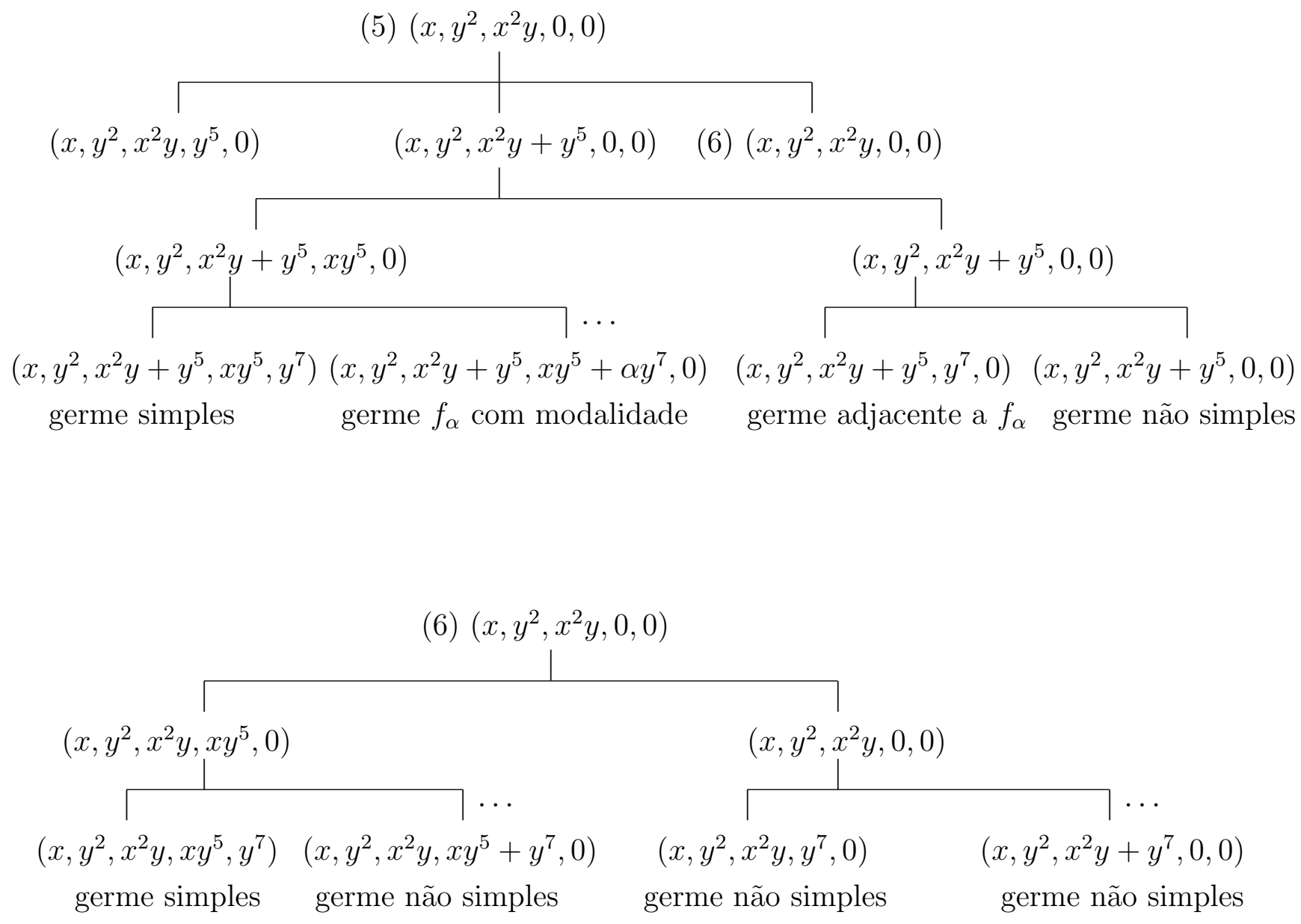

(3) $\left(x, y^{2}, 0,0,0\right)$

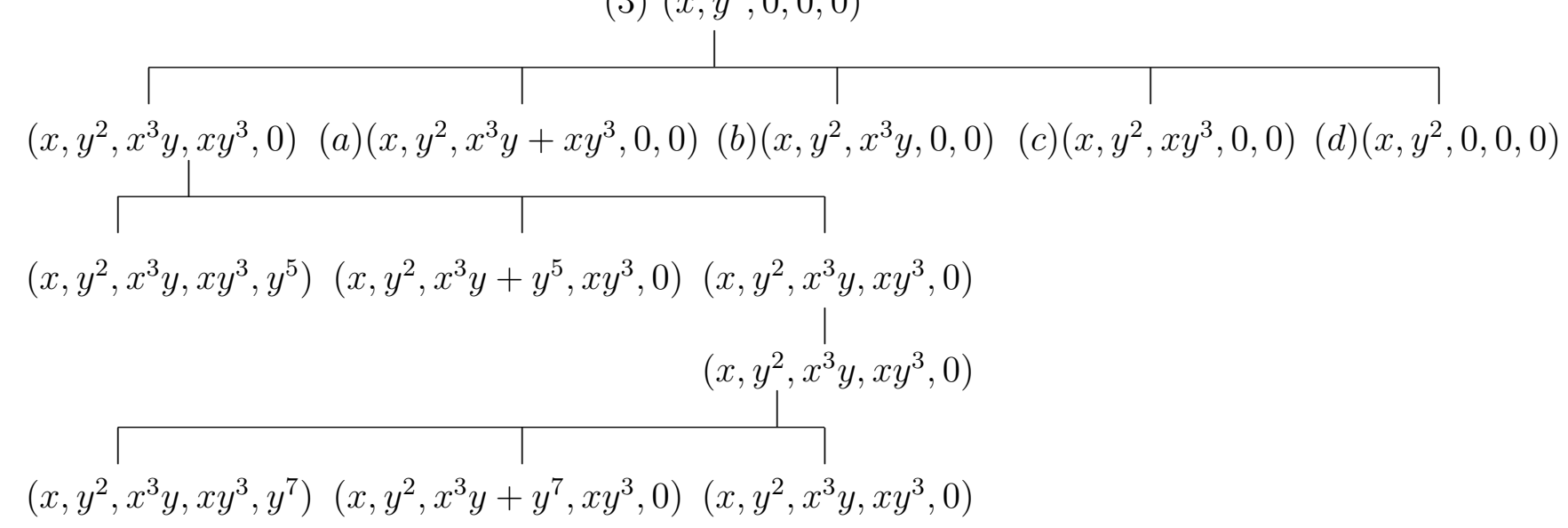

germe simples germe não simples germe não simples 

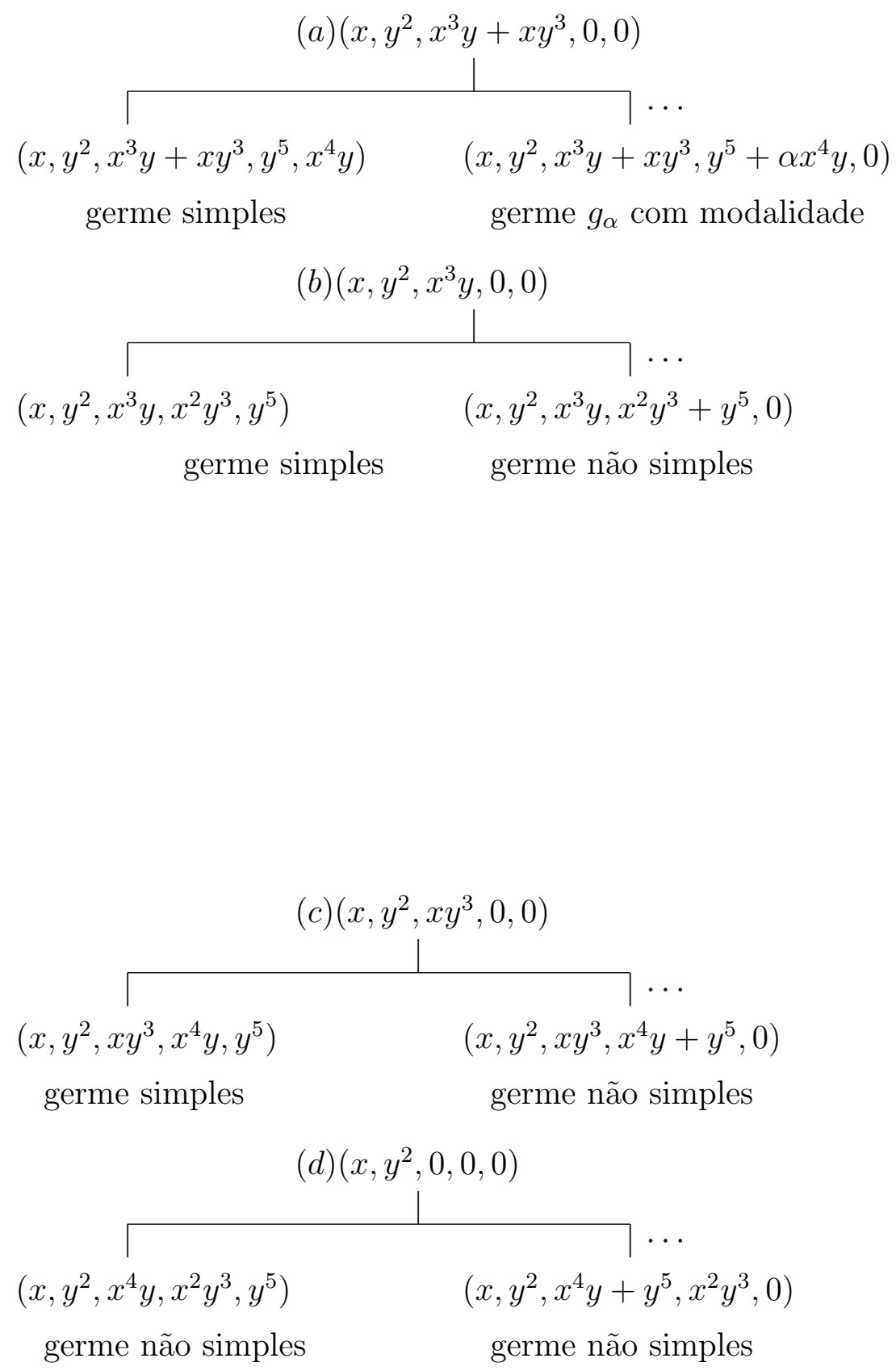

Obtemos também a lista dos germes $\mathcal{A}$-simples de $\mathbb{C}^{2}$ em $\mathbb{C}^{6}$, multiplicidade 2, usando o Método da Tranversal Completa. Esta lista consiste dos germes $(f, 0)$, onde $f$ é um germe simples de $\mathbb{C}^{2}$ em $\mathbb{C}^{5}$. 
Teorema 5.1.8 Todo germe $\mathcal{A}$-simples $f:\left(\mathbb{C}^{2}, 0\right) \rightarrow\left(\mathbb{C}^{6}, 0\right)$, de multiplicidade 2 , é $\mathcal{A}$-equivalente a um dos seguintes germes:

$$
\begin{aligned}
& \left(x, x y, y^{2}, y^{2 k+1}, 0,0\right), k \geq 1 \\
& \left(x, y^{2}, y^{3}, x^{k} y, 0,0\right), k \geq 2 \\
& \left(x, y^{2}, y^{3}+x^{k} y, x^{l} y, 0,0\right), l>k \geq 2 \\
& \left(x, y^{2}, x^{2} y+y^{2 k+1}, x y^{3}, 0,0\right), k \geq 2 \\
& \left(x, y^{2}, x^{2} y, x y^{3}, y^{2 k+1}, 0\right), k \geq 2 \\
& \left(x, y^{2}, x^{2} y, y^{5}, 0,0\right) \\
& \left(x, y^{2}, x^{2} y+y^{5}, x y^{5}, y^{7}, 0\right) \\
& \left(x, y^{2}, x^{2} y, x y^{5}, y^{7}, 0\right) \\
& \left(x, y^{2}, x^{3} y+y^{5}, x y^{3}, 0,0\right) \\
& \left(x, y^{2}, x^{3} y, x y^{3}, y^{5}, 0\right) \\
& \left(x, y^{2}, x^{3} y, x y^{3}, y^{7}, 0\right) \\
& \left(x, y^{2}, x^{3} y+x y^{3}, y^{5}, x^{4} y, 0\right) \\
& \left(x, y^{2}, x^{3} y, x^{2} y^{3}, y^{5}, 0\right) \\
& \left(x, y^{2}, x y^{3}, x^{4} y, y^{5}, 0\right)
\end{aligned}
$$

Além disso, fazendo a clasificação para $n \geq 6$, multiplicidade 2, obtemos apenas germes $\mathcal{A}$-simples do tipo $(f, 0)$, onde $f:\left(\mathbb{C}^{2}, 0\right) \rightarrow\left(\mathbb{C}^{n-1}, 0\right)$ é um germe $\mathcal{A}$-simples de multiplicidade 2.

Teorema 5.1.9 Todo germe $\mathcal{A}$-simples $f:\left(\mathbb{C}^{2}, 0\right) \rightarrow\left(\mathbb{C}^{n}, 0\right), n \geq 6$, de multiplicidade 2, é $\mathcal{A}$-equivalente a um germe do tipo

$$
(f, \underbrace{0, \ldots, 0}_{n-5 \text { vezes }})
$$

onde $f:\left(\mathbb{C}^{2}, 0\right) \rightarrow\left(\mathbb{C}^{5}, 0\right)$ é um germe simples.

Consideramos agora os germes $\mathcal{A}$-simples de $\mathbb{C}^{2}$ em $\mathbb{C}^{5}$, com multiplicidade 3. Temos o seguinte: 
Teorema 5.1.10 Todo germe $\mathcal{A}$-simples $f:\left(\mathbb{C}^{2}, 0\right) \rightarrow\left(\mathbb{C}^{5}, 0\right)$, de multiplicidade 3 , é $\mathcal{A}$ equivalente a um germe do tipo

$$
\begin{aligned}
& \left(x, x y, x y^{2}, y^{3}, y^{3 k+2}+y^{3 \ell+1}\right), 1<k<\ell<2 k \\
& \left(x, x y, x y^{2}, y^{3}, y^{3 k+2}\right), k \geq 1 \\
& \left(x, x y, x y^{2}+y^{3 k+2}, y^{3}, y^{3 m+1}+y^{3 \ell+2}\right), 1<k<m \leq \ell<2 k \\
& \left(x, x y, x y^{2}+y^{3 k+2}, y^{3}, y^{3 m+1}\right), 1 \leq k<m \leq 2 k \\
& \left(x, x y, x y^{2}+y^{3 k+2}, y^{3}, 0\right), k \geq 1 \\
& \left(x, x y, x y^{2}+y^{3 k+2}, y^{3}, y^{3 m+2}+y^{3 \ell+1}\right), 1<k<m<\ell \leq 2 k \\
& \left(x, x y, x y^{2}+y^{3 k+2}, y^{3}, y^{3 m+2}\right), 1<k<m<2 k \\
& \left(x, x y+y^{3 k+2}, x y^{2}, y^{3}, y^{3 \ell+1}+y^{3 m+2}\right), 1 \leq k<\ell \leq m \leq 2 k \\
& \left(x, x y+y^{3 k+2}, x y^{2}, y^{3}, y^{3 \ell+1}\right), 1 \leq k<\ell \leq 2 k \\
& \left(x, x y+y^{3 k+2}, x y^{2}, y^{3}, y^{3 \ell+2}+y^{3 m+1}\right), 1<k<\ell<m \leq 2 k \\
& \left(x, x y+y^{3 k+2}, x y^{2}, y^{3}, y^{3 \ell+2}\right), 1 \leq k<\ell \leq 2 k \\
& \left(x, x y+y^{3 k+2}, x y^{2}, y^{3}, 0\right), k \geq 1 \\
& \left(x, x y, x y^{2}, y^{3}, y^{3 k+1}+y^{3 \ell+2}\right), 1<k \leq \ell<2 k-1 \\
& \left(x, x y, x y^{2}, y^{3}, y^{3 k+1}\right), k \geq 1 \\
& \left(x, x y, x y^{2}+y^{3 k+1}, y^{3}, y^{3 m+2}+y^{3 \ell+1}\right), 1<k \leq m<\ell<2 k \\
& \left(x, x y, x y^{2}+y^{3 k+1}, y^{3}, y^{3 m+2}\right), 1 \leq k \leq m<2 k \\
& \left(x, x y, x y^{2}+y^{3 k+1}, y^{3}, y^{3 \ell+1}+y^{3 m+2}\right), 1<k<\ell \leq m<2 k \\
& \left(x, x y, x y^{2}+y^{3 k+1}, y^{3}, y^{3 \ell+1}\right), 1<k<\ell<2 k \\
& \left(x, x y, x y^{2}+y^{3 k+1}, y^{3}, 0\right), k \geq 1 \\
& \left(x, x y, y^{3}, y^{4}, y^{5}\right) \\
& \left(x, x y, y^{3}, y^{4}, 0\right) \\
& \left(x, x y, y^{3}, y^{5}, y^{7}\right) \\
& \left(x, x y, y^{3}, y^{5}, 0\right) \\
& \left(x, x y+y^{3}, x y^{2}, y^{4}, y^{2 k+1}\right), k \geq 2 \\
& \left(x, x y+y^{3}, x y^{2}+y^{2 k+1}, y^{4}, y^{2 k+3}\right), k \geq 2 \\
& \left(x, x y+y^{3}, x y^{2}+y^{2 k+1}, y^{4}, 0\right), k \geq 2 \\
& \left(x, x y, x y^{2}, y^{3}, y^{4}\right) \\
& \left(x, x y+y^{4}, x y^{2}, y^{3}, y^{5}\right) \\
& \left(x, x^{2} y+y^{4}, x y^{2}, y^{3}, x y^{4}\right) \\
& \left(x, x^{2} y+y^{4}+y^{5}, x y^{2}, y^{3}, 0\right) \\
& \left(x, x^{2} y+y^{4}, x y^{2}, y^{3}, 0\right) \\
&
\end{aligned}
$$

Neste caso faremos apenas algumas observações, omitindo o processo de classificação.

Lema 5.1.11 As $\mathcal{A}^{3}$-órbitas de germes simples de multiplicidade 3 são equivalentes a um dos seguintes germes:
1. $\left(x, x y, x y^{2}, y^{3}, 0\right)$
2. $\left(x, x y, y^{3}, 0,0\right)$
3. $\left(x, x y+y^{3}, x y^{2}, 0,0\right)$
4. $\left(x, x y+y^{3}, 0,0,0\right)$ 


\section{Demonstração:}

Em [18], vimos que um germes simples de multiplicidade 3 tem 2-jato equivalente a $(x, x y, 0,0,0)$. Seja $T \subset H^{3}(2,5)$ satisfazendo

$$
\mathcal{M}_{2}^{3} \mathcal{O}_{2,5} \subset T \mathcal{A}_{1} f+T+\mathcal{M}_{2}^{4} \mathcal{O}_{2,5}
$$

onde

$$
T \mathcal{A}_{1} f=\mathcal{M}_{2}^{2}\left\{\left[\begin{array}{l}
1 \\
y \\
0 \\
0 \\
0
\end{array}\right],\left[\begin{array}{l}
0 \\
x \\
0 \\
0 \\
0
\end{array}\right]\right\}+f^{*} \mathcal{M}_{5}^{2}\left\{e_{1}, \ldots, e_{5}\right\}
$$

Logo, $T=\left\{x y^{2} \cdot e_{i}, i=3,4,5, y^{3} \cdot e_{j}, \quad j=2,3,4,5\right\}$. Então o 3 -jato de $f$ é equivalente a

$$
\left(x, x y+b_{0} y^{3}, a_{1} x y^{2}+b_{1} y^{3}, a_{2} x y^{2}+b_{2} y^{3}, a_{3} x y^{2}+b_{3} y^{3}\right) .
$$

Se $b_{i} \neq 0$, para algum $i=1,2,3$, digamos $b_{3} \neq 0$ removemos o termo $y^{3}$ das coordenadas $e_{2}, e_{3}$ e $e_{4}$ e obtemos, após mudanças apropriadas, $\left(x, x y, \overline{a_{1}} x^{2} y, \overline{a_{2}} x y^{2}, a_{3} x y^{2}+b_{3} y^{3}\right)$.

Se $\overline{a_{i}} \neq 0$, para algum $i=1,2$, obtemos $\left(x, x y, x y^{2}, y^{3}, 0\right)$.

Se $\overline{a_{i}}=0$, para $i=1,2$, obtemos $\left(x, x y, x y^{2}+y^{3}, 0,0\right)$.

Se $\overline{a_{i}}=0$, para $i=1,2$, e $a_{3}=0$, obtemos $\left(x, x y, y^{3}, 0,0\right)$, que é equivalente ao germe $\left(x, x y, x y^{2}+y^{3}, 0,0\right)$. Use a mudança de coordenada na fonte $y \mapsto y-c x$.

Suponhamos agora que $b_{0} \neq 0$. Então removemos o termo $y^{3}$ das coordenadas $e_{3}, e_{4}$ e $e_{5}$ e obtemos, após mudanças apropriadas, $\left(x, x y+y^{3}, x^{2} y, 0,0\right)$ ou $\left(x, x y+y^{3}, 0,0,0\right)$. 
Seja $\left(x, x y, x y^{2}, y^{3}, 0\right) \in J^{3 k+1}(2,5), k \geq 1$. Usando o método da Transversal Completa vamos obter o seguinte diagrama:

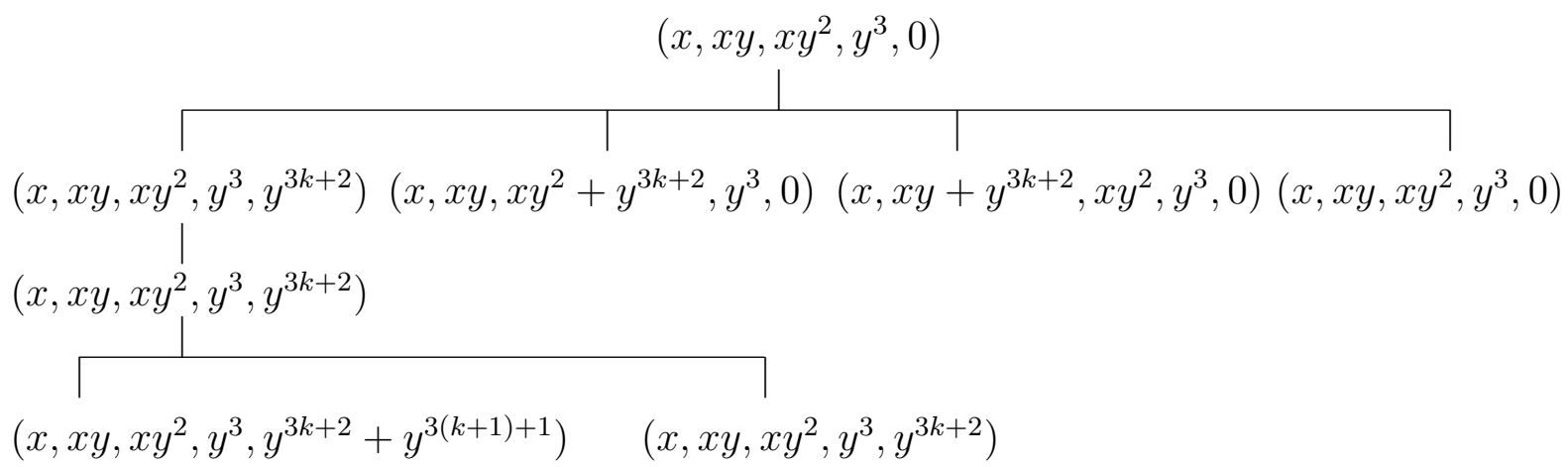

Seja $\left(x, x y, x y^{2}, y^{3}, y^{3 k+2}\right) \in J^{3 l}(2,5), l>1$. Usando o método da Transversal Completa vamos obter $T \subset H^{3 l+1}(2,5)$ tal que

$$
\mathcal{M}_{2}^{3 l+1} \mathcal{O}_{2,5} \subset T \mathcal{A}_{1} f+T+\mathcal{M}_{2}^{3 l+2} \mathcal{O}_{2,5}
$$

onde $\mathcal{M}_{2}^{3 l+1}=\left\langle x^{3 l+1}, x^{3 l} y, \ldots, x y^{3 l}, y^{3 l+1}\right\rangle \mathrm{e}$

$$
T \mathcal{A}_{1} f=\mathcal{M}_{2}^{2}\left\{\left[\begin{array}{c}
1 \\
y \\
y^{2} \\
0 \\
0
\end{array}\right],\left[\begin{array}{c}
0 \\
x \\
2 x y \\
3 y^{2} \\
(3 k+2) y^{3 k+1}
\end{array}\right]\right\}+f^{*}\left(\mathcal{M}_{5}^{2} \mathcal{O}_{5,5}\right)
$$

Logo, $T=\left\{y^{3 l+1}\left(e_{5}\right)\right\}$ se $l<2 k$ e $T=\emptyset$, se $l \geq 2 k$. E obtemos as seguintes séries: $\left(x, x y, x y^{2}, y^{3}, y^{3 k+2}+y^{3 l+1}\right), 1<k<l<2 k$, e $\left(x, x y, x y^{2}, y^{3}, y^{3 k+2}\right), k \geq 1$.

No caso de germes de multiplicidade 3 , quando fazemos a classificação dos germes de $\left(\mathbb{C}^{2}, 0\right)$ em $\left(\mathbb{C}^{6}, 0\right)$, aparecem novos germes simples (não apenas os do tipo $(f, 0)$, com $f$ aparecendo no Teorema 5.1.10). Joachim H. Rieger conjectura que os novos germes são, além daqueles do tipo $(f, 0)$, os que adicionam monônios com filtração maior (quando a $f$ na dimensão anterior é $\mathcal{A}$ - simples) e os que separam monônios de $f$ (neste caso $f$ pode ser não simples). Esta conjectura é válida quando comparamos a classificação obtida por Klotz, Pop e Rieger em [18] de $\left(\mathbb{C}^{2}, 0\right)$ em $\left(\mathbb{C}^{4}, 0\right)$ e a classificação obtida para o caso de $\left(\mathbb{C}^{2}, 0\right)$ em $\left(\mathbb{C}^{5}, 0\right)$ neste trabalho. 


\section{Referências Bibliográficas}

[1] V.I. Arnol'd, Simple singularities of curves, Proc. Steklov Inst. Math. 226 (1999), 20-28.

[2] M.F.Atiyah, I.G.MacDonald, Introduction to Commutative Algebra, Addison-Wesley, 1969.

[3] G.F. Barbosa and J.H. Rieger, Relations between A-invariants of equidimensional corank-1 maps, submitted to Amer. J. Math..

[4] J.W. Bruce and T. Gaffney, Simple singularities of mappings from $\mathbb{C}$ to $\mathbb{C}^{2}$, J. London Math. Soc. 24 (1982), 465-474.

[5] W. Bruns and U. Vetter, Determinantal Rings, Springer LNM 1327, Springer Verlag 1988.

[6] J. Damon, Finite determinacy and topological triviality I., Invent. Math. 62 (1980), 299324.

[7] J. Damon and D. Mond, $\mathcal{A}$-codimension and vanishing topology of discriminants, Invent. Math. 106 (1991), 217-242.

[8] T. Fukuda and G. Ishikawa, On the number of cusps of stable perturbations of a planeto-plane singularity, Tokyo J. Math. 10 (1987), 375-384.

[9] T. Fukui, J.J. Nuno Ballesteros and M.J. Saia, On the number of singularities in generic deformations of map germs, J. London Math. Soc. 58 (1998), 141-153.

[10] T. Gaffney, Polar multiplicities and equisingularity of map germs, Topology 32 (1993), 185-223.

[11] T. Gaffney and D. Mond, Cusps and double folds of germs of analytic maps $\mathbb{C}^{2} \rightarrow \mathbb{C}^{2}, J$. London Math. Soc. 43 (1991), 185-192.

[12] C.G. Gibson, Singular points of smooth mappings, Research Notes in Mathematics, 25, Pitman, 1979.

[13] C.G. Gibson and C.A. Hobbs, Simple singularities of space curves, Math. Proc. Camb. Phil. Soc. 113 (1993), 297-310. 
[14] V.V. Goryunov, Semi-simplicial resolutions and homology of images and discriminants of mappings, Proc. London Math. Soc. 70 (1995), 363-385.

[15] G.M. Greuel, Der Gauß-Manin-Zusammenhang isolierter Singularitäten von vollständigen Durchschnitten, Math. Ann. 241 (1975), 235-266.

[16] C.A. Hobbs and N.P. Kirk, On the classification and bifurcation of multigerms of maps from surfaces to 3-space, Math. Scand. 89 (2001), 57-96.

[17] K. Houston, Calculating generalised image and discriminant Milnor numbers in low dimensions, Glasgow Math. J. 43 (2001), 165-175.

[18] C. Klotz, O. Pop and J.H. Rieger, Real double-points of deformations of $\mathcal{A}$-simple mapgerms from $\mathbb{R}^{n}$ to $\mathbb{R}^{2 n}$, Math. Proc. Camb. Phil. Soc., 142 (2007), 341-363.

[19] D.T. Lê, Calculation of Milnor number of isolated singularity of complete intersection, Functional Anal. Appl. 8 (1974), 127-131.

[20] E.J.N. Looijenga, Isolated singular points on complete intersections, London Math. Soc. Lect. Note Series 77, Cambridge University Press 1984.

[21] W.L. Marar and D. Mond, Multiple point schemes for corank 1 maps, J. London Math. Soc. 39 (1989), 553-567.

[22] W.L. Marar, J.A. Montaldi and M.A.S. Ruas, Multiplicities of zero-schemes in quasihomogeneous corank-1 singularities $\mathbb{C}^{n} \rightarrow \mathbb{C}^{n}$, in: Singularity Theory (Liverpool, 1996), London Math. Soc. Lect. Note Series 263, Cambridge University Press 1999, 353-367.

[23] J.Milnor, Singular Points of Complex Hypersurfaces, Annals of Mathematics Studies, 61, Princeton University Press, 1968.

[24] D. Mond, On the classification of germs of maps from $\mathbb{R}^{2}$ to $\mathbb{R}^{3}$, Proc. London Math. Soc. 50 (1985), 333-369.

[25] J.H. Rieger, Families of maps from the plane to the plane, J. London Math. Soc. 36 (1987), 351-369.

[26] J.H. Rieger, Invariants of equidimensional corank-1 maps, Banach Center Publ., Vol. 36 (2004), 239-248.

[27] J.H. Rieger, Relations betweem $\mathcal{A}$-invariants of semi-quasihomogeneous, equidimensional corank-1 maps, Unpublished Notes.

[28] J.H. Rieger, $\mathcal{A}$-unimodal map-germs into the plane, Hokkaido Math. J. 33 (2004), 47-64. 
[29] J.H. Rieger and M.A.S. Ruas, Classification of $\mathcal{A}$-simple germs from $k^{n}$ to $k^{2}$, Compositio Math. 79 (1991), 99-108.

[30] J.H. Rieger and M.A.S. Ruas, $M$-deformations of $\mathcal{A}$-simple $\sum^{n-p+1}$ germs from $\mathbb{R}^{n}$ to $\mathbb{R}^{p}, n \geq p$, Math. Proc. Camb. Phil. Soc. 139 (2005), 333-349.

[31] C.T.C. Wall, Finite determinancy of smooth map-germs, Bull. London Math. Soc. 13 (1981), 481-539.

[32] R.G. Wik-Atique, On the classification of multigerms of maps from $\mathbb{C}^{2} \rightarrow \mathbb{C}^{3}$ under $\mathcal{A}$ equivalence, Real and Complex Singularities (J. V. Bruce and F. Tari, eds.), Proceedings of the 5th Workshop on Real and Complex Singularities, São Carlos, Brazil, (1998), 119-133. 